CERN-TH/98-206

OUTP-98-48P

hep-th/9809033

\title{
On Stringy Thresholds in SYM/AdS Thermodynamics
}

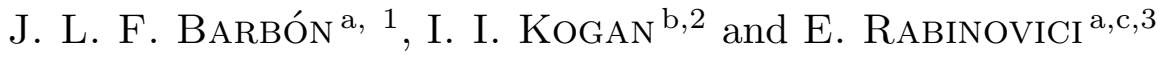 \\ a Theory Division, CERN \\ CH-1211, Geneva 23, Switzerland \\ b Theoretical Physics, Department of Physics \\ 1 Keble Road, Oxford, OX1 3NP, UK \\ ${ }^{\mathrm{c}}$ Racah Institute of Physics \\ The Hebrew University \\ Jerusalem 91904, Israel
}

We consider aspects of the role of stringy scales and Hagedorn temperatures in the correspondence between various field theories and AdS-type spaces. The boundary theory is set on a toroidal world-volume to enable small scales to appear in the supergravity backgrounds also for low field-theory temperatures. We find that thermodynamical considerations tend to favour background manifolds with no string-size characteristic scales. The gravitational dynamics censors the reliable exposure of Hagedorn physics on the supergravity side, and the system does not allow the study of the Hagedorn scale by low-temperature field theories. These results are obtained following some heuristic assumptions on the character of stringy modifications to the gravitational backgrounds. A rich phenomenology appears on the supergravity side, with different string backgrounds dominating in different regions, which should have field-theoretic consequences. Six-dimensional world volumes turn out to be borderline cases from several points of view. For lower dimensional world-volumes, a fully holographic behaviour is exhibited to order $1 / N^{2}$, and open strings in their presence are found to have a thermodynamic Hagedorn behaviour similar to that of closed strings in flat space.

$09 / 98$

1 barbon@mail.cern.ch, ${ }^{2}$ i.kogan@physics.ox.ac.uk, ${ }^{3}$ eliezer@vxcern.cern.ch 


\section{Introduction}

The suggestion of a correspondence between a class of effective conformal field theories and special, anti- de Sitter (AdS), string backgrounds, [1], has allowed the study of some basic properties of gauge theories in the strong coupling limit2. One may also attempt to turn the tables around and investigate the possibility that the correspondence sheds some light on outstanding problems in gravity and string theory. We would like to investigate the extent to which physics at small (i.e. string) distance scales emerging on the supergravity side can be understood in terms of gauge dynamics. One such issue is the nature of the Hagedorn transition. We will report here on a search for an answer to that question. Actually, something has already been learned about the nature of gravity at the very first stage of setting up the correspondence. The correspondence can work only if the theory of gravity is allowed to contain at the same time configurations of different topologies. This is vindicated by studying thermodynamical aspects of the correspondence. The thermodynamics on the Conformal Field Theory side, (CFT), was studied in [5], and [6], [7], on the basis of the large $N$ CFT/AdS conjectured correspondence. For strong coupling the CFT thermodynamics is obtained by evaluating the gravitational thermodynamics in an AdS background. When several masterfields may contribute on the supergravity side, one is instructed to take all of them into account, [6], and this is needed to fully acount for thermodynamical properties of the CFT.

In particular, when the spatial world-volume of the four-dimensional gauge theory has the topology of $\mathbf{S}^{3}$, there are two possible bulk manifolds ending on the same worldvolume boundary. One is an AdS-like vacuum which we shall denote $X_{\mathrm{vac}}$, while the other is a black-hole like manifold denoted $X_{\mathrm{bh}}$. Both are taken into account in calculations performed on the supergravity side. The transition between them at some finite temperature expresses a first-order phase transition following from the occurence of a sort of gravitational collapse, along the lines of [8]. Technically, it is based on the balance of the gravitational free energy between the vacuum AdS space with a radiation thermal ensemble defined on it, or an AdS black hole in equilibrium with the radiation at the same temperature. Such a transition is indeed of first-order in the large $N$ limit (the classical limit from the gravity point of view), and it has been established for the case of spherical topology. The feature of several masterfields contributing has been responsible for the

${ }^{2}$ Earlier work in this direction includes [2], [3], [4]. 
holographic [9] nature of the mapping at the classical [6], [7], and one-loop level, [10]. It was also the gravitational manifestation of (as well as the motivation to search for) a large $N$ phase transition at finite sphere volume in gauge theory. It will turn out that it also influences the extent to which one may probe the nature of the Hagedorn transition with these methods.

The above large $N$ phase transition is not driven by the emergence of light states involved in the onset of the Hagedorn phenomena. In order to search for such states one needs to first address two issues. The first concerns the identification of the possibility of the emergence of light string states (states which could lead to a Hagedorn instability) by analysing a given supergravity background. These we expect to occur whenever such a manifold has a string-scale circumference in the Euclidean time direction. The second concerns the estimate of the gauge theory temperature, dual to a given supergravity configuration. The length of the euclidean time circumference depends in general on the value of the spatial coordinates. We estimate the dual gauge theory temperature by considering the circumference at those points for which the radial AdS coordinate is of the scale appropriate to the AdS curvature (which is equal to the scale of the complementary compact manifold). From this point of view a search [10] for those states in the case of $\mathbf{S}^{3}$ topology has indicated the existence of a 'Hagedorn censorship'. The manifold $X_{\mathrm{vac}}$ is found to contain non-contractible thermal loops whose length is of the order of the string scale, $\ell_{s} \sim \sqrt{\alpha^{\prime}}$, only when the temperature of the gauge theory itself is of order

$$
T_{\text {gauge }}=R^{-1}\left(g_{s} N\right)^{1 / 4}=1 / \ell_{s}
$$

where $g_{s}$ is proportional to the string coupling, and $R$, the radius of the spatial $\mathbf{S}^{3}$, was set at $\ell_{s}\left(g_{s} N\right)^{1 / 4}$ to estimate the gauge theory temperature.

However, at temperatures of order $T_{c} \sim R^{-1}$, much smaller than (1.1) in the supergravity regime $g_{s} N \gg 1$, the manifold $X_{\mathrm{bh}}$ takes over the thermodynamical description and its horizon shields any string-length non-contractible thermal loops. This inability of the gauge theory to allow a peek into the Hagedorn regime we have termed Hagedorn censorship. In principle, string theory should serve as our guide to what the exact correspondence is and to its possible limitations, [11]. As long as this is not resolved in general, we explore several possibilities. The first is that the correspondence is valid at string scale temperatures. In that case one could be learning something about the string theory, i.e. that there is no Hagedorn transition in this string background, perhaps as a result of a 
decay and collapse of the towers of excited states. Another possibility, which we check in this paper, is that the Hagedorn transition may be exposed if the world volume of the boundary gauge theory is modified so that the supergravity background geometries contain string scales also for low gauge theory temperatures. We also probe the possibility that at string scales the corresponding systems change so as to allow for the expression of string scales on both sides. A rather rich phenomenology will unfold and at the same time the theory will continue to resist devulging reliable information on a Hagedorn transition.

In section 2 we choose the topology of the world volume so that small scales are present also for low gauge theory temperatures. This is done by setting up the gauge theory in a different spatial topology: a three-torus $\mathbf{T}^{3}$. Manifolds corresponding to the $X_{\mathrm{vac}}$ and $X_{\mathrm{bh}}$ ones defined for the $\mathbf{S}^{3}$ topology exist also for this case (see [12, and references therein, for more complicated topolgies in the case of two-dimensional horizons). The manifolds are similar in some ways but not identical, in particular $X_{\mathrm{bh}}$ is well defined for this topology at all temperatures and non-contractible thermal (as well as spatial) loops of string-scale length are present in $X_{\mathrm{vac}}$ for arbitrarily low gauge-theory temperatures. This would thus seem an appropriate arena in which to attempt to circumvent the Hagedorn censorship. The discussion can be carried out also for more general $\mathbf{T}^{p}$ spatial manifolds, obtained from the world-volume of $D p$-branes in Type-II string theory. In this more general case the CFT/AdS correspondence is really a SYM/SUGRA correspondence, between the, generically non-conformal $\mathcal{N}=4$ super-Yang-Mills theories on the $(p+1)$-dimensional world-volumes, and the appropriate scaled up throat geometries, as in [13]. We also keep the original, non blown-up, black brane configurations for further use.

In section 3 the classical free energy is evaluated for the various classical manifolds without yet taking into account the neccesity to modify the manifolds at small scales. The classical dominance of the manifold $X_{\mathrm{bh}}$ is obtained. The evaluation of the corresponding specific heat offers a first glimpse at the phenomenon of lack of bulk decoupling for $p \geq 5$. In section 4 the impact of higher derivative operators coming from integrating out massive string modes in the bulk of the holographic manifold is considered. Indications are found that the gauge theory is self-contained and exhibits no extra stringy scale. In section 5 the Hagedorn censorship and its limitations are established. This is done by estimating the modifications needed to reflect the singular nature of the vacuum manifolds at the string scale, both in gauge theory and string theory language. In the process, situations in which Hagedorn scales may be exposed are discussed. In particular we mostly study the 
SYM/SUGRA correspondence in dimension $(p+1)$ within a specific limiting procedure: the large $N$ expansion of the full string-brane theory is taken first, and each term in this expansion is then expanded in the limit $N \rightarrow \infty$ with $g_{s} N=$ fixed, the supergravity regime corresponds to $g_{s} N \gg 1$. For $g_{s} N \ll 1$ one is on much less firm ground. String corrections are of order one, curvatures are large and the classical supergravity picture gets fuzzy. Nevertheless, as it is there that Hagedorn features surface, we do venture into that region.

The SYM/SUGRA correspondence actually may intrinsically set its own limitations. On the gauge theory side, the description ceases to be well defined in the absence of more ultra-violet data for $p \geq 5$, whereas the gravitational side shows unstable thermodynamics, with negative values of the specific heat in the same range of dimensions. This would indicate a breakdown of the correspondence. It turns out that for $p \geq 5$ this is indeed the case, the gravitational systems have only regions of negative specific heat at the leading (planar gauge or classical gravitational) order. At the next to leading order holography breaks down for the same values of $p$, and the extensive ten-dimensional behaviour is restored. This gives an additional point of view from which it seems that modifications

of the correspondence are imminent for these large values of $p$ [14]. In section 6 this breakdown of holography for $p \geq 5$, is demonstrated on the basis of the $1 / N$ corrections to the free energy. Having dealt with the Hagedorn regime from the SYM/SUGRA point of view we conclude by studying that region in section 7 , from a more directly stringy point of view, this is done both by applying the analysis of section 5 to background configurations which are not fully blown-up and by studying the system now at weak coupling. In the first case the Hagedorn transition emerges, but it does so on both sides of the correspondence. In the second situation $p=5$ is recaptured as a critical dimension and open strings in the presence of branes behave thermodynamically not so different than closed strings.

\section{Classical Throat Geometries}

In this section we discuss the two types of bulk manifolds whose boundary is $\mathbf{S}^{1} \times \mathbf{T}^{p}$, with $p<7$. The cases $p \geq 7$ cannot be realized in terms of asymptotically flat brane solutions, and we do not consider them. 


\subsection{Vacuum Manifold}

The construction of the vacuum manifold, denoted $X_{\mathrm{vac}}$, starts from the closed-string massless backgrounds around the euclidean extremal $D p$-brane with toroidal topology $\mathbf{S}_{\beta}^{1} \times \mathbf{T}_{L}^{p}$, 15. In terms of the harmonic function

$$
H=1+\left(\frac{b}{r}\right)^{7-p}
$$

we have the following complete geometry, which we shall denote by $E D p$ :

$$
d s^{2}(E D p)=\frac{1}{\sqrt{H}}\left(d \tau^{2}+d \vec{y}^{2}\right)+\sqrt{H}\left(d r^{2}+r^{2} d \Omega_{8-p}^{2}\right) .
$$

In addition, we also have profiles for the dilaton and the Ramond-Ramond (RR) $(8-p)$ form with $N$ units of flux through the angular sphere $\mathbf{S}^{8-p}$ :

$$
\begin{aligned}
& e^{-2 \phi}=e^{-2 \phi_{\infty}} H^{\frac{p-3}{2}} \\
& F_{r \tau \vec{y}}=e^{-\phi_{\infty}} F_{r \tau \vec{y}}^{\prime}=e^{-\phi_{\infty}} \partial_{r} \frac{1}{H} .
\end{aligned}
$$

The torus identifications are $\tau \equiv \tau+\beta$ and $\vec{y} \equiv \vec{y}+L$. The 'charge radius' is defined by

$$
b \equiv \ell_{s}\left(g_{s} N\right)^{\frac{1}{7-p}} .
$$

The precise relation between $g_{s}$ and the asymptotic string coupling is

$$
g_{s}=d_{p}(2 \pi)^{p-2} e^{\phi_{\infty}}=e^{\phi_{\infty}} \frac{(2 \pi)^{7-p}}{\operatorname{Vol}\left(\mathbf{S}^{8-p}\right)} .
$$

The 'gauge theory' scaling takes $\ell_{s} \rightarrow 0$ and $r \rightarrow 0$, holding the energy scale $u=r / \ell_{s}^{2}$ fixed. This corresponds, in the microscopic D-brane picture, to the decoupling limit of open-string excited modes, while keeping fixed the massless Higgs expectation values (i.e. the moduli space) of the low energy SYM theory. In the geometry (2.2), this limit blows-up the throat of the extremal $D p$-brane solution, to obtain a new manifold, denoted $X_{\mathrm{vac}}$, [1], [13]:

$$
\frac{d s^{2}\left(X_{\mathrm{vac}}\right)}{\ell_{s}^{2}}=\frac{u^{\frac{7-p}{2}}}{g_{\mathrm{YM}} \sqrt{d_{p} N}}\left(d \tau^{2}+d \vec{y}^{2}\right)+\frac{g_{\mathrm{YM}} \sqrt{d_{p} N}}{u^{\frac{7-p}{2}}} d u^{2}+g_{\mathrm{YM}} \sqrt{d_{p} N} u^{\frac{p-3}{2}} d \Omega_{8-p}^{2},
$$

where the Yang-Mills coupling is defined by

$$
g_{\mathrm{YM}} \sqrt{d_{p} N}=\ell_{s}^{\frac{p-3}{2}} \sqrt{g_{s} N} .
$$


It should be noted that only the fully blown-up manifolds are known for the case of CFT on a sphere, as in [6], [7]. A brane realization incorporating the spherical geometry of the world-volume, and allowing a smooth transition into flat ten- or eleven-dimensional Minkowski space-time, is not yet available.

Restoring the radial variable $r=u \ell_{s}^{2}$, this limit is the near-horizon scaling, consisting of neglecting the 1 in the harmonic function $H$ :

$$
d s^{2}\left(X_{\mathrm{vac}}\right)=\frac{r^{2}}{b_{r}^{2}}\left(d \tau^{2}+d \vec{y}^{2}\right)+\frac{b_{r}^{2}}{r^{2}} d r^{2}+b_{r}^{2} d \Omega_{8-p}^{2},
$$

where we have defined the quantity:

$$
b_{r}^{2} \equiv r^{\frac{p-3}{2}} b^{\frac{7-p}{2}} .
$$

In this way the metric is cast in the form of a 'product' $A d S_{p+2} \times \mathbf{S}^{8-p}$, both factors with a varying radius of curvature, equal to $b_{r}$. Thus the $\alpha^{\prime}$ expansion parameter, i.e. the scalar curvature in string units is given by

$$
\alpha^{\prime} R \sim\left(\frac{\ell_{s}}{b_{r}}\right)^{2} .
$$

For $p>3$ the curvature is small as $r \rightarrow \infty$ and large as $r \rightarrow 0$, and the other way around for $p<3$. The critical point where the curvature becomes of order one in string units is at

$$
r_{c} \sim \ell_{s}\left(g_{s} N\right)^{\frac{1}{3-p}} \sim b\left(g_{s} N\right)^{\frac{4}{(7-p)(3-p)}} .
$$

The supergravity description (closed strings propagating in a smooth, 'large' background) will be good at large coordinate radius for $p>3$, and near the horizon for $p<3$. In order for the smooth region to be large in string units we require $g_{s} N \gg 1$. As pointed out in [13], this is in nice agreement with the idea that the radial coordinate represents (via the Higgs expectation values of a probe $D$-brane) the renormalization group scale in the gauge theory language. In this dictionary, the ultraviolet region is the upper part of the throat, and the infrared is represented by the near-horizon region. Thus, the breakdown of the supergravity description in the ultraviolet for $p<3$ is in correspondence with the asymptotic freedom (i.e. perturbative nature) of SYM at short distances, in less than four dimensions. Conversely, the breakdown of the supergravity description in the infrared for $p>3$ agrees with the non-renormalizability of SYM above four dimensions; i.e. these 
theories flow to free (perturbative) fixed points in the infrared. In other words, there is a complementarity between the SYM and SUGRA descriptions, with a cross-over roughly located at $r \sim r_{c}$, where the geometry becomes 'fuzzy'.

There is also a non-perturbative threshold for $p \neq 3$, due to the fact that the dilaton has a non-trivial profile. At a radial coordinate of order

$$
r_{g} \sim r_{c} N^{\frac{4}{(7-p)(p-3)}}
$$

the throat develops local strong coupling $e^{\phi\left(r_{g}\right)} \sim \mathcal{O}(1)$. At these points, lying in the ultraviolet (infrared) for $p>(<) 3$, we must go over dual descriptions, as pointed out in [13]. In the present paper, we take the large $N$ limit before any other expansion, so that $N$ is larger than any dimensionless parameter formed out of couplings or ratios of scales in the problem. In particular, we take $N \gg g_{s} N$, and then we can always postpone the non-perturbative threshold, as compared to other thresholds which depend only on the combination $g_{s} N$.

One such threshold that will interest us in this paper is the onset of $\alpha^{\prime}$ corrections due to light states with support in the background geometry under consideration. In particular, light string winding modes will set in when the proper length of the non-contractible circles is of order the string scale. For termal winding modes this happens at radial coodinates

$$
r_{\beta}=b\left(\frac{\ell_{s}}{\beta}\right)^{\frac{4}{7-p}}=\frac{b}{\left(g_{s} N\right)^{\frac{4}{(7-p)^{2}}}}\left(\frac{b}{\beta}\right)^{\frac{4}{7-p}}
$$

and we also have a threshold for spatial winding modes, at $r_{L}$ defined by substituting $\beta$ by $L$ in this equation. Below these radii, the semiclassical propagation of Type-II strings is weighted in perturbation theory by the effective expansion parameter $\left(g_{s} / m \ell_{s}\right)^{2}$ for each closed string loop. The mass $m<1 / \ell_{s}$ corresponds to the new light winding modes. This means that string perturbation theory breaks down, even if we are in a region where the nominal value of the local string coupling is small, $e^{\phi(r)} \sim N^{-1}$, for large, but finite, $N \gg g_{s} N \gg 1$.

In the following, it will be important to distinguish between the blown-up throat manifold (2.5) or (2.7), denoted $X_{\mathrm{vac}}$, and the complete $D p$-brane geometry in (2.2), denoted $E D p$. The meaning of the radial coordinates $r>b$ in $X_{\mathrm{vac}}$ is associated to the ultraviolet behaviour of the system in the field theory limit (2.5), which involves, in particular, the decoupling of the string oscillators. There are, however, indications that ([16], [17]) 
keeping the end of throat or 'neck' at $r \sim b$, is associated to the stringy thresholds in the D-brane sector. In the following, we will make explicit this distinction between the full system, with the stringy thresholds kept in place, and the truncated system: SYM at weak coupling, and (infinite) throat geometry at strong coupling.

A crucial difference between these throat geometries with flat boundary space-time and the time slicing adequate for finite spherical topology in [6], is the occurrence of arbitrarily small non-contractible loops down the throat. This means that, for example, Hagedorn scales occur down the throat of $X_{\text {vac }}$ no matter how small we dial the ratio $L / \beta$ at the boundary.

\subsection{Black-Hole Manifold}

The $X_{\text {bh }}$ or black-hole-type manifold, with the same asymptotics $\mathbf{S}_{\beta}^{1} \times \mathbf{T}_{L}^{p}$ is obtained from the blow-up of the throat of the near-extremal black $D p$-brane. We denote this manifold by $B D p$, with metric [15]

$$
d s^{2}(B D p)=\frac{1}{\sqrt{H}}\left(h d \tau^{2}+d \vec{y}^{2}\right)+\sqrt{H}\left(\frac{d r^{2}}{h}+r^{2} d \Omega_{8-p}^{2}\right),
$$

where we have introduced the Schwarzschild-like harmonic function:

$$
h=1-\left(\frac{r_{0}}{r}\right)^{7-p},
$$

and the function $H$ also depends on $r_{0}$ :

$$
\begin{aligned}
H & =1+\left(\frac{r_{p}}{r}\right)^{7-p} \\
r_{p}^{7-p} & =-\frac{r_{0}^{7-p}}{2}+\left(b^{2(7-p)}+\frac{r_{0}^{2(7-p)}}{4}\right)^{1 / 2} .
\end{aligned}
$$

The dilaton and Ramond-Ramond (RR) backgrounds have the same form as in (2.2), with $H$ defined in (2.15), so that the extremal solution is the limit $r_{0} \rightarrow 0$ of the black-brane solution (2.13). The extremality parameter $r_{0}$ gives the location of the horizon. In the euclidean section, spacetime is restricted to $r \geq r_{0}$.

The gauge theory regime is defined by the near-horizon limit, i.e. by the restriction $r, r_{0} \ll b$, and we obtain the scaled-up throat, or $X_{\mathrm{bh}}$ manifold as

$$
d s^{2}\left(X_{\mathrm{bh}}\right)=\frac{r^{2}}{b_{r}^{2}}\left(\left(1-r_{0}^{7-p} / r^{7-p}\right) d \tau^{2}+d \vec{y}^{2}\right)+\frac{b_{r}^{2}}{r^{2}} \frac{d r^{2}}{\left(1-r_{0}^{7-p} / r^{7-p}\right)}+b_{r}^{2} d \Omega_{8-p}^{2} .
$$




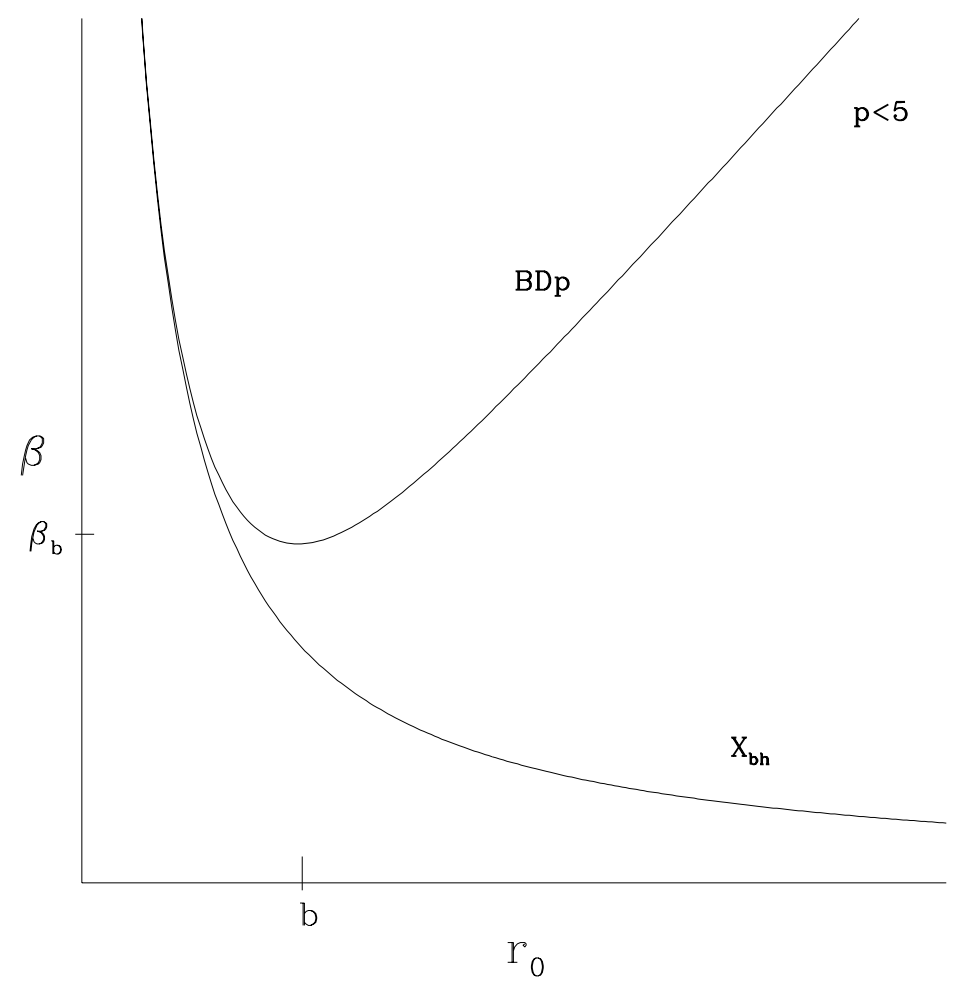

Fig. 1: Inverse Hawking temperature as a function of $r_{0}$ for the $p<5$ manifolds.

The euclidean section (2.15), or (2.16) is smooth at $r=r_{0}$ only if the variable

$$
\theta=\frac{\tau}{2} \sqrt{\frac{\partial_{r} g_{\tau \tau}}{\partial_{r} g_{r r}}}
$$

has period $2 \pi$. So, if the period of $\tau$ is $\beta$, we obtain

$$
\beta=4 \pi \sqrt{\frac{\partial_{r} g_{r r}\left(r_{0}\right)}{\partial_{r} g_{\tau \tau}\left(r_{0}\right)}}=\frac{4 \pi}{7-p} r_{0} \sqrt{H\left(r_{0}\right)} .
$$

This equation defines the canonical thermal ensemble, providing the functional dependence of the mass as a function of temperature. It applies not only to the scaled up throat manifold $X_{\mathrm{bh}}$ in (2.16), but also to the complete black-brane geometry, $B D p$ in (2.13).

The behaviour is rather different from the case of spherical boundary, studied in [7], where the black-hole manifold $X_{\mathrm{bh}}$ could only be defined for sufficiently large temperature: $\beta_{0}<b$, with $\beta_{0}^{-1}$ the temperature at the origin of the AdS space. For the range of allowed temperatures, there was a branch of large black holes with radius $r_{0}>b$ and positive 
specific heat, along with a brach of small ones $r_{0}<b$, and negative specific heat. The canonical ensemble was dominated at high temperature $\beta_{0}<b$ by the large black holes with positive specific heat.

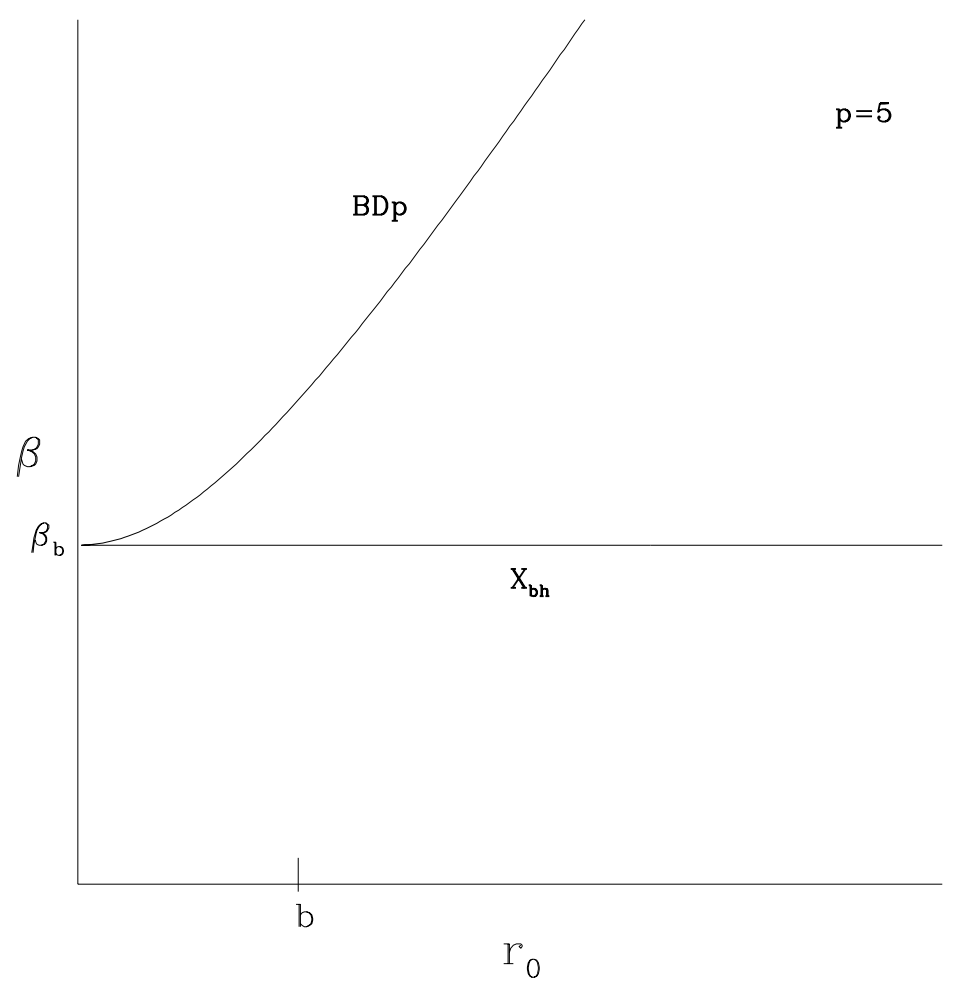

Fig. 2: Inverse Hawking temperature as a function of $r_{0}$ for the $p=5$ manifolds.

For most values of $p$, the situation is exactly opposite in the case of the $B D p$ metric. For $p<5$, the complete curve (2.18) has a minimum at radius $r_{0} \sim b$. It grows without bound in the near-extremal or gauge theory regime $r_{0} \ll b$, with asymptotics $\beta\left(r_{0} \ll\right.$ $b) \sim b\left(b / r_{0}\right)^{\frac{5-p}{2}}$. This is precisely the temperature-horizon relation for the scaled up $X_{\mathrm{bh}}$ manifold:

$$
\beta\left(X_{\mathrm{bh}}\right)=\frac{4 \pi}{7-p} b\left(\frac{b}{r_{0}}\right)^{\frac{5-p}{2}} .
$$

This is a branch with positive specific heat, the one that can be related to gauge theory thermodynamics. In the complete $B D p$ metric, there is also the usual Schwarzschild regime, $r_{0} \gg b$, with the law (universal for all values of $p$ ):

$$
\beta\left(r_{0} \gg b\right) \sim r_{0}
$$


and negative specific heat. We shall see that this branch (which is absent in $X_{\mathrm{bh}}$ ) is thermodynamically unfavoured in the canonical ensemble. Thus, $B D p(p<5)$ can only be defined for sufficiently low temperatures $T<T_{b} \sim b^{-1}$, and the branch with positive specific heat $r_{0} \ll b$, is defined for arbitrarily low temperatures, unlike the case of spherical boundary, (see fig. 1.)

For $p=5$, a marginal case, $\beta\left(r_{0}\right)$ is a monotonously increasing function with vanishing derivative at $r_{0}=0$. The metric $B D p(p=5)$ is defined only for temperatures $T<T_{b} \sim$ $b^{-1}$, and has negative specific heat for all temperatures. The scaled-up throat $X_{\mathrm{bh}}$ has constant temperature, independent of the value of $r_{0}, T=1 / 2 \pi b$, and thus formally has infinite specific heat (fig. 2.)

The last case, $p=6$, shows a similar behaviour. There is a single regime of the complete black-brane metric $B D p(p=6)$ with negative specific heat, but now defined for arbitrary high temperatures. The scaled-up throat $X_{\mathrm{bh}}(p=6)$ has also negative specific heat and is only defined for very high temperatures, with asymptotics given by eq. (2.19), which still applies (fig. 3.)

We see that it is unlikely that $X_{\mathrm{bh}}$ is related to gauge theory thermodynamics in the cases $p=5,6$, since they lack a phase with positive specific heat at low temperatures.

The local red-shifted temperature in $X_{\mathrm{bh}}$ diverges at the horizon. This is a general property of any horizon, and it would lead us to suspect that a Hagedorn regime is reached when this local red-shifted temperature is of order the string scale. On the other hand, the topology of the thermal modes in $X_{\mathrm{bh}}$ is trivial. What happens is that the 'Hagedorn' region at the tip of $X_{\mathrm{bh}}$ is defined by a disc with a proper radius of the order of the string length. Therefore, a thermal winding mode in the local Hagedorn region can unwind at no cost of energy. Indeed, string 'propagation' is completely smooth around the horizon of the euclidean $X_{\mathrm{bh}}$ manifold. Hagedorn effects should be taken into account when metastable light winding modes can be identified. This would happen for example if the local temperature in $X_{\mathrm{bh}}$ reaches the string scale at large radial distance from the horizon, so that it costs a large amount of energy to 'unwind' the thermal 'winding' modes. In this case the geometry of $X_{\mathrm{bh}}$ is that of a very 'thin cigar' and this can be arranged only when the asymptotic temperature itself is of the order of the string scale. 


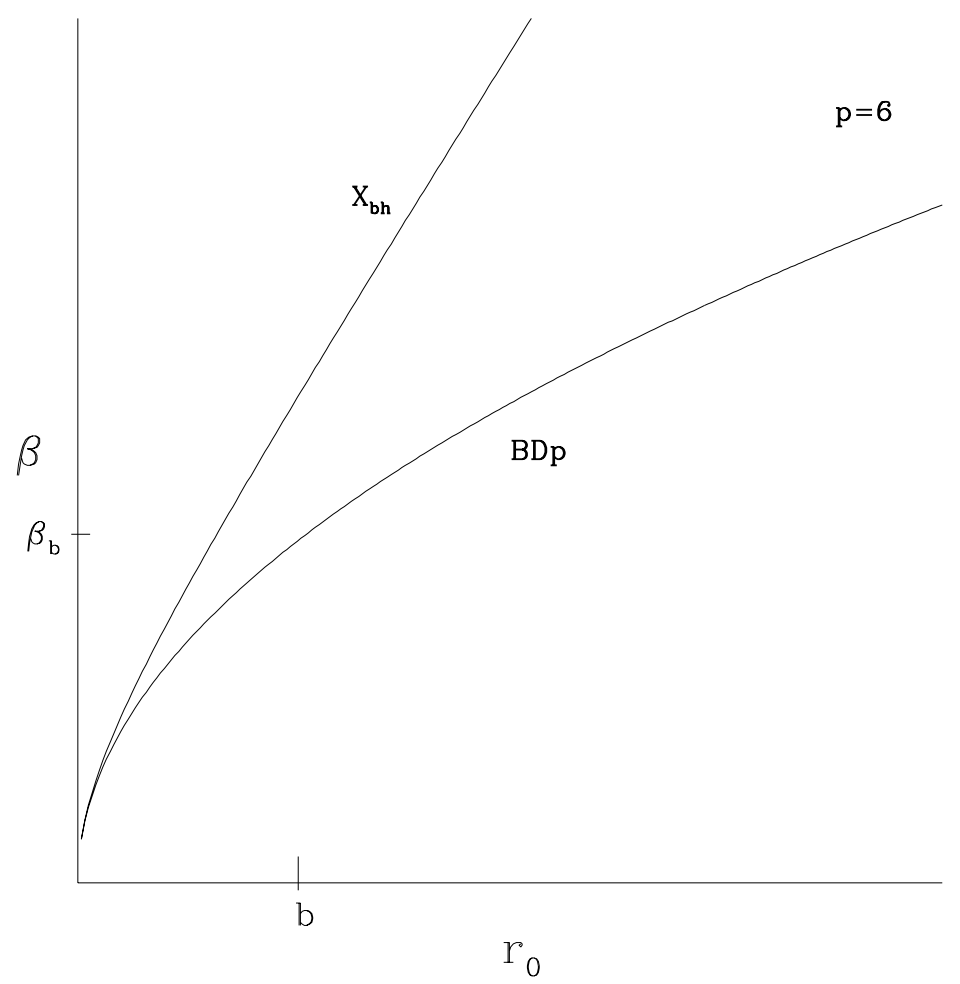

Fig. 3: Inverse Hawking temperature as a function of $r_{0}$ for the $p=6$ manifolds.

\section{Classical Free Energy and Black-Hole Dominance}

For a boundary of topology $\mathbf{S}^{1} \times \mathbf{S}^{3}$, the bulk gravitational theory exhibited a phase transition at some critical temperature [6], [7]. Here we repeat these considerations for the case at hand. The analysis in this section is based on the classical supergravity approximation, as in [18], although we have argued in section 1 that it should become invalid sufficiently far down the throat of $X_{\mathrm{vac}}$. Such effects will be analyzed separately in section 5 .

The action, (or planar free energy in the gauge theory interpretation), of any of the manifolds under consideration is

$$
I(X)_{c \ell}=\beta M(X)-S(X) .
$$

We shall consider first the cases of the general metrics, $E D p$ and $B D p$, given by (2.2) and (2.13), respectively, and then recover the actions of the scaled throat geometries $X_{\mathrm{vac}}$ and $X_{\text {bh }}$ by taking appropriate near-horizon limits. 
In formula (3.1), $M$ is the ADM mass, derived from the asymptotics of the $d=10-p$ black hole obtained upon dimensional reduction in the wrapped $\vec{y}$-directions [19]:

$$
g_{\tau \tau} \sim 1-\frac{16 \pi G_{d} M}{(d-2) \operatorname{Vol}\left(\mathbf{S}^{d-2}\right) r^{d-3}},
$$

with $G_{d}=G / L^{p}$ the $d=10-p$ dimensional Newton constant, and $G \equiv G_{d=10}=$ $8 \pi^{6} \alpha^{\prime 4} e^{2 \phi_{\infty}}$. The $d$-dimensional metric is obtained by dimensional reduction of (2.15) and subsequent rescaling to the Einstein frame:

$$
d s^{2} \rightarrow e^{\frac{-4\left(\phi_{d}-\phi_{\infty}\right)}{d-2}} d s^{2},
$$

with $\phi_{d}$ the $d$-dimensional dilaton:

$$
e^{-2 \phi_{d}}=e^{-2 \phi} \sqrt{\operatorname{det}\left(g_{\vec{y} \vec{y}}\right)} .
$$

The resulting Einstein-frame black-hole metric is

$$
d s_{d}^{2}=H^{\frac{3-d}{d-2}} h d \tau^{2}+H^{\frac{1}{d-2}}\left(d r^{2} / h+r^{2} d \Omega_{d-2}^{2}\right),
$$

and matching the asymptotics to (3.2) one obtains

$$
M(B D p)=\frac{\operatorname{Vol}\left(\mathbf{S}^{8-p}\right) L^{p}}{16 \pi G}\left((8-p) r_{0}^{7-p}+(7-p) r_{p}^{7-p}\right) .
$$

There are two limiting regimes: the Schwarzschild regime, where the horizon radius is much larger than the charge radius $r_{0} \gg b$,

$$
M\left(r_{0} \gg b\right)_{B D p} \sim(8-p) \frac{\operatorname{Vol}\left(\mathbf{S}^{8-p}\right) L^{p}}{16 \pi G} r_{0}^{7-p},
$$

and the opposite, near-extremal regime:

$$
M\left(r_{0} \ll b\right)_{B D p} \sim M_{E D p}+\frac{9-p}{2} \frac{\operatorname{Vol}\left(\mathbf{S}^{8-p}\right) L^{p}}{16 \pi G} r_{0}^{7-p},
$$

with

$$
M_{E D p}=(7-p) \frac{\operatorname{Vol}\left(\mathbf{S}^{8-p}\right) L^{p}}{16 \pi G} b^{7-p}
$$

the mass of the extremal $D p$-brane system. The corresponding 'mass' of the 'throats' $X_{\text {vac }}$ and $X_{\mathrm{bh}}$ is defined by these near-extremal limits

$$
\begin{aligned}
M\left(X_{\mathrm{vac}}\right) & =M_{E D p} \\
M\left(X_{\mathrm{bh}}\right) & =M_{E D p}+\frac{9-p}{2} \frac{\operatorname{Vol}\left(\mathbf{S}^{8-p}\right) L^{p}}{16 \pi G} r_{0}^{7-p} .
\end{aligned}
$$


The entropy can be obtained in the standard fashion from (3.5):

$$
S=\frac{A_{H}}{4 G_{d}}=\frac{\operatorname{Vol}\left(\mathbf{S}^{8-p}\right) L^{p}}{4 G} r_{0}^{8-p} \sqrt{H\left(r_{0}\right)}
$$

or, using the temperature formula (2.18):

$$
S=(7-p) \frac{\operatorname{Vol}\left(\mathbf{S}^{8-p}\right) L^{p}}{16 \pi G} \beta r_{0}^{7-p} .
$$

Written in this way, this formula for the entropy is exact and applies to all cases $B D p$ and $X_{\mathrm{bh}}$. The entropy of the 'vacuum' manifolds $E D p$ and $X_{\text {vac }}$ is obtained as the $r_{0} \rightarrow 0$ limit of this expression and it vanishes identically.

By definition, the thermodynamics of the manifolds $X_{\mathrm{vac}}, X_{\mathrm{bh}}$ is equivalent to the one of $E D p, B D p$ in the gauge theory regime, i.e. for temperatures $T \ll T_{b} \sim b^{-1}$. We first focus on the analysis of the field-theory limit thermodynamics and postpone to section 7 the discussion of the other Schwarzschild-like regimes of the $B D p, E D p$ pairs.

The free energy of $X_{\mathrm{bh}}$, obtained through (3.1), is

$$
I\left(X_{\mathrm{bh}}\right)_{c \ell}=-\frac{5-p}{2} \frac{\operatorname{Vol}\left(\mathbf{S}^{8-p}\right) L^{p}}{16 \pi G} \beta r_{0}^{7-p}+\beta M_{E D p} .
$$

The action of $X_{\mathrm{vac}}$ follows by subtituting $r_{0}=0$ in this formula. One finds that it is completely given by a vacuum energy 3

$$
I\left(X_{\mathrm{vac}}\right)_{c \ell}=\beta M_{E D p}
$$

Therefore, the action difference between $X_{\mathrm{vac}}$ and $X_{\mathrm{bh}}$, or the planar free energies, becomes

$$
\beta F(\beta)_{\mathrm{planar}}=I\left(X_{\mathrm{bh}}\right)_{c \ell}-I\left(X_{\mathrm{vac}}\right)_{c \ell}=-N^{2}(5-p) C_{0}\left(g_{s} N\right)^{\frac{2(p-3)}{7-p}} L^{p} b^{\gamma-p} \beta^{-\gamma},
$$

with $C_{0}$ the positive constant,

$$
C_{0}=\frac{(2 \pi)^{7-2 p}}{2 \operatorname{Vol}\left(\mathbf{S}^{8-p}\right)}\left(\frac{4 \pi}{7-p}\right)^{\frac{2(7-p)}{5-p}}
$$

3 By using in (3.1) the ADM definition of mass to calculate the classical action, we bypass subtleties in the treatment of boundary terms at infinity, as in [7]. Such subtleties arise if we evaluate directly the canonical action on the solution, and amount to the correct prescription of (3.1), with $M$ the ADM mass, and $S$ the Bekenstein-Hawking entropy (3.11). 
and $\gamma$ the critical exponent

$$
\gamma=\frac{9-p}{5-p}
$$

This critical exponent is larger than that of free fields $\gamma_{\text {free }}=p$, for all $p$ except the conformal one $p=3$ where $\gamma=\gamma_{\text {free }}$.

The thermodynamical balance in (3.15) yields $X_{\mathrm{bh}}$ dominance for $p<5$ at all temperatures. This contrasts with the behaviour with spherical boundary topology, [6], [7], where $X_{\mathrm{vac}}$ dominated the low-temperature phase. Thus, within this approximation, there is no large $N$ 'flop' between $X_{\mathrm{vac}}$ and $X_{\mathrm{bh}}$, as long as as $p<5$.

For $p=5$ we have a marginal behaviour, with exactly balanced free energies between $X_{\mathrm{vac}}$ and $X_{\mathrm{bh}}$ at the temperature at which $X_{\mathrm{bh}}$ is defined, $T\left(X_{\mathrm{bh}}\right)=1 / 2 \pi b$. However, for any other temperature, only $X_{\mathrm{vac}}$ contributes to the canonical ensemble. Thus, generically, planar thermodynamics is dominated by vacuum energy for $p=5$.

The situation is analogous for $p=6$. In this case, the vacuum-subtracted free energy of $X_{\mathrm{bh}}$ is positive, and therefore thermodynamically suppressed. $X_{\mathrm{vac}}$ dominates at all temperatures.

This concludes our discussion of the purely classical thermodynamical properties of the blown-up manifolds $X_{\mathrm{vac}}$ and $X_{\mathrm{bh}}$. We find a neat difference in the canonical ensemble, depending on whether $p$ is larger or smaller than five but, for a given dimension, we $d o$ not find $\mathbf{T}^{p}$ analogues of the finite-volume phase transition reported in [6], [7] for the spherical case. In the rest of the paper we investigate to what extent these conclusions are modified by stringy effects, either in the bulk of the $X$-manifolds, or perhaps localized around singular loci.

\section{Short-Distance Corrections}

According to the dictionary developed in [1], the $\alpha^{\prime}$ or low-energy expansion of the string theory in the AdS space corresponds to the strong-coupling expansion of the large $N$ gauge theory in inverse powers of the 't Hooft coupling: $\alpha^{\prime} / b^{2}=1 / \sqrt{g_{\mathrm{YM}}^{2} N}$. On the other hand, string-loop corrections yield the topological expansion of the gauge theory diagrams: $g_{s}^{2}=\left(g_{\mathrm{YM}}^{2} N / N\right)^{2}$, and semiclassical Yang-Mills instanton corrections are related to Dinstanton processes in the Type-IIB theory [20], [21], [22]. In the low-energy approximation, the effect of the massive string states propagating in the throat geometries $X_{\mathrm{vac}}, X_{\mathrm{bh}}$, can 
be incorporated into the low-energy supergravity action by means of higher-derivative operators in the massless fields, suppressed by powers of the string length squared $\alpha^{\prime}=\ell_{s}^{2}$. The form of the effective action induced within string perturbation theory is

$$
I_{\mathrm{eff}} \sim \frac{1}{g_{s}^{2}} \sum_{g \geq 0} \sum_{n \geq 1}\left(g_{s}^{2}\right)^{g}\left(\alpha^{\prime}\right)^{n} \int \frac{d \mathrm{Vol}}{\alpha^{\prime 5}} \mathcal{O}_{(g, n)}
$$

If only the fields in (2.2) and (2.3) are excited, the important operators of engineering dimensions $\operatorname{dim}(\mathcal{O})=2 n=2\left(n_{c}+n_{\phi}+n_{f}\right)$, generated at $g$-loop order, take the form

$$
\mathcal{O}_{\left(g, n_{i}\right)} \sim\left(e^{-2\left(\phi-\phi_{\infty}\right)}\right)^{1-g} R^{n_{c}}(\partial \phi)^{2 n_{\phi}}\left|F_{p+2}^{\prime}\right|^{2 n_{f}}
$$

where $R$ stands for the Riemann tensor or some contraction thereof. The classical values $\left\langle\mathcal{O}_{(g, n)}\right\rangle_{X}$ on the manifold $X$ determine the leading corrections in first-order perturbation theory in the coefficient of such operators. A proper treatment of the $g_{s}$ and $\alpha^{\prime}$ expansion to $k$-th order should also include the effect of the operators (4.2), through the back-reaction on the manifold geometry, to order $k-1$. However, for the purposes of the qualitative dependence on the various dimensionful parameters, like for example the temperature, we can estimate the effects of (4.1) by its expectation value on the large $N$ uncorrected saddle point $I_{\text {eff }} \sim \sum \int\left\langle\mathcal{O}_{\text {eff }}\right\rangle_{X}$, provided we expand all expressions in power series in $g_{s}$ and $\alpha^{\prime}$.

Under these circumstances, we can estimate the effects of these short-distance corrections through scaling arguments (for a precise calculation of a leading correction in the $p=3$ case, see 23.) We consider the black-hole manifold $X_{\mathrm{bh}}$, and recover $X_{\mathrm{vac}}$ as the $r_{0} \rightarrow 0$ limit. It is useful to introduce yet another system of coordinates:

$$
z^{2} \equiv b_{r}^{2}=r^{\frac{p-3}{2}} b^{\frac{7-p}{2}}
$$

so that, for $p \neq 3, r^{2} / b_{r}^{2}=z^{\alpha} / b^{\alpha}$ with $\alpha=2(7-p) /(p-3)$. Define also

$$
(t, \vec{x}) \equiv b^{-\alpha / 2}(\tau, \vec{y})
$$

thereby casting the near-extremal metric (2.16) in the form

$$
d s^{2}\left(X_{\mathrm{bh}}\right)=z^{\alpha}\left(\left(1-z_{0}^{2 \alpha} / z^{2 \alpha}\right) d t^{2}+d \vec{x}^{2}\right)+\frac{C d z^{2}}{\left(1-z_{0}^{2 \alpha} / z^{2 \alpha}\right)}+z^{2} d \Omega_{8-p}^{2}
$$

with $C=16 /(p-3)^{2}$. This representation, without explicit dependence on $b$ or $\alpha^{\prime}$, is useful for scaling arguments, because a local invariant of dimension $D$ takes the form 
$z^{-D} f\left(z_{0}^{2 \alpha} / z^{2 \alpha}\right)$. For example, $D=2$ for the curvature scalar $R$ or the dilaton gradient squared $(\partial \phi)^{2}$. In addition, translational invariance with respect to the $(t, \vec{x})$ coordinates ensures the effective action is proportional to $\beta_{t} L_{x}^{p}=b^{-\alpha(p+1) / 2} \beta L^{p}$.

We then find the following near-horizon asymptotics for the relevant invariants contributing to the effective action:

$$
\begin{aligned}
e^{-2 \phi} & \sim \frac{1}{g_{s}^{2}}\left(\frac{b}{z}\right)^{2(7-p)} \\
\left|F_{p+2}\right|^{2} & \sim \frac{1}{z^{2}} e^{-2 \phi} \\
R & \sim \frac{1}{z^{2}} .
\end{aligned}
$$

The overall scaling of each operator in (4.2) depends only on the total $\alpha^{\prime}$ counting index $n=n_{c}+n_{\phi}+n_{f}$. Thus, a general operator of dimension $2 n$, generated at $g$ loop order, has the following scaling when evaluated on the solution (4.5):

$$
\left\langle\mathcal{O}_{g, n}\right\rangle_{X_{\mathrm{bh}}} \sim N^{2-2 g} \frac{\left(g_{s} N\right)^{\frac{10-2 n}{7-p}}}{\left(g_{s} N\right)^{2-2 g}} \beta L^{p} b^{B}\left[z^{A} f\left(z^{2 \alpha} / z_{0}^{2 \alpha}\right)\right]_{z_{-}}^{z_{+}}
$$

where $z_{ \pm}$denote the limits between which the radial coordinate is integrated. The power of $b$ is

$$
B=2 n-10+2(7-p)(1-g)-\frac{\alpha}{2}(p+1),
$$

and $A$ is fixed accordingly by dimensional analysis. Restoring the $r$ variable through (4.3), we find the following result for the effective action:

$$
I_{\mathrm{eff}}=\beta E_{\mathrm{vac}}+N^{2} \sum_{g=0}^{\infty} \sum_{n=1}^{\infty} I_{g, n}\left(g_{s} N\right)^{\frac{2-2 n}{7-p}}\left(\frac{g_{s} N}{N}\right)^{2 g},
$$

where

$$
I_{g, n}=-C_{g, n}\left(g_{s} N\right)^{\frac{2(p-3)}{7-p}} L^{p} b^{\gamma_{g, n}-p} \beta^{-\gamma_{g, n}}
$$

and the critical exponent is:

$$
\gamma_{g, n}=\frac{9-p}{5-p}-\frac{p-3}{5-p}(n-1-g(7-p))=\frac{(3-p) n+g\left(10 p-p^{2}-21\right)+6}{5-p} .
$$

The classical term is obtained for $n=1$ and $g=0$. The estimate of the short-distance corrections to the $X_{\text {vac }}$ free energy can be done along the same lines, putting $r_{0}=0$ in 
the solution, and evaluating (4.7) between two limiting radii $r_{ \pm}$. The result is clearly proportional to $\beta$, and we can naturally interpret it as a correction to the vacuum energy.

A very interesting feature of $(4.9)$ and $(4.10)$ is the possibility of eliminating the separate dependence on $g_{s} N$ and $b$. One can combine the dependence on the effective D-brane string coupling and the 'transmuted' string scale, $b$, into the single dimensionful 't Hooft coupling (2.6):

$$
I_{\mathrm{eff}}=\beta E_{\mathrm{vac}}-\sum_{g=0}^{\infty} \sum_{n=1}^{\infty} N^{2-2 g} B_{g, n}\left(g_{\mathrm{YM}}^{2} N\right)^{\frac{\gamma_{g, n}-p}{p-3}} L^{p} \beta^{-\gamma_{g, n}} .
$$

This result shows how, at least within the low-energy expansion, the effects of closedstring excited states have a gauge theory interpretation, i.e. the only intrinsic scale of the system is the dimensionful Yang-Mills coupling $g_{\mathrm{YM}}^{2} N$, and the type-IIB string in the blown-up $X$-manifold 'behaves' like a genuine 'QCD-string'. This seems to be a non-trivial property of the fully blown-up throats $X_{\mathrm{vac}}$ and $X_{\mathrm{bh}}$. We shall argue in section 7 that the transmutation of the $\alpha^{\prime}$ dependence into gauge theory parameters is not complete for the non-blown-up manifolds $E D p$ and $B D p$.

The scaling laws in (4.12) admit some generalizations. For example, one may turn on boundary values for other fields in the supergravity backgrounds, corresponding to turning on more operators in the gauge theory. Special care is needed then for the RR potentials, which have $\mathcal{O}\left(N^{0}\right)$ scaling in the large $N$ limit. One such example would be the leading large $N$ contribution to the theta angle dependence in the four-dimensional theory considered in [24]. Such contributions to the effective action scale like a $g=1, n=1$ term, even if they should be considered as 'planar' from the point of view of the 't Hooft expansion.

The scaling law for the conformal case $p=3$ can be obtained by direct subtitution of $z=b$ in (4.6). One gets the same answer by directly plugging $p=3$ in the final formula (4.11). The index $\gamma$ does not receive any loop or $\alpha^{\prime}$ corrections, i.e. $\gamma_{g, n}=\gamma_{0,1}=3$.

An interesting remark concerns the large order behaviour of $\gamma_{g, n}$. We find that, at fixed $g$,

$$
\lim _{n \rightarrow \infty} \gamma_{g, n} \rightarrow+\infty \text { if } p<3 \text { or } p>5 .
$$

Since $\gamma$ is a measure of the effective dimension for massless field excitations in the large temperature regime, $\gamma_{g, n} \rightarrow \infty$ can be interpreted as a bad high temperature behaviour, in 
the sense that the theory exhibits a host of new degrees of freedom at high energies. This is compatible with the fact that for $p<3$, the supergravity description becomes inappropriate in the ultraviolet, where D-brane perturbation theory takes over. The pathology at high temperature for $p>5$ is to be expected in the light of our previous discussion about the lack of gravity decoupling for $p \geq 5$. We also find

$$
\lim _{n \rightarrow \infty} \gamma_{g, n}=-\infty \text { if } 3<p<5
$$

This corresponds to the fact that the ultraviolet is well described by the supergravity in this range of brane dimensions, and therefore high order corrections in $\alpha^{\prime}$ are less and less singular in the high-temperature limit.

On the other hand, the large-order behaviour with respect to string-loop corrections works the other way around. At fixed $n, \gamma \rightarrow-\infty$ for $p \neq 4$. But $\gamma \rightarrow+\infty$ for $p=4$. The interpretation of this could be that, precisely for $p=4$, the local value of the string coupling grows up the throat, towards the ultraviolet.

\section{Effects of Light Winding Modes}

Having dealt in the previous section with short-distance effects coming from the massive closed string states propagating in the bulk of $X_{\mathrm{vac}}, X_{\mathrm{bh}}$, we now turn to other limitations of the supergravity description. Namely, with branes of topology $\mathbf{S}^{1} \times \mathbf{T}^{p}$, both $X_{\mathrm{vac}}$ and $X_{\mathrm{bh}}$ support non-contractible cycles. In particular, $r=0$ is singular for $X_{\mathrm{vac}}$ because these non-contractible cycles shrink to zero size. As pointed out in section 2, at

radial coordinates of order $r_{\beta}$, temporal cycles have string length, while spatial cycles do so at the point

$$
r_{L}=b\left(\frac{\ell_{s}}{L}\right)^{\frac{4}{7-p}}=\frac{b}{\left(g_{s} N\right)^{\frac{4}{(7-p)^{2}}}}\left(\frac{b}{L}\right)^{\frac{4}{7-p}} .
$$

Accordingly, we expect important modifications of the geometry at these scales. At high temperatures from the point of view of the gauge theory, $\beta \ll L$, the corrections to the geometry implied by the light thermal winding modes dominate, since $r_{\beta} \gg r_{L}$ in this regime. On the other hand, at low temperatures $\beta \gg L$, effects due to light spatial winding modes dominate. 


\subsection{Spatial Winding Modes and Finite-Size Effects}

In the low temperature regime, $\beta \gg L$, the approximate supersymmetry allows us to determine the effective geometry at radial distances $r \ll r_{L}$. In this region, spatial winding modes on the $\mathbf{T}^{p}$ torus are lighter than momentum modes. In we want to continue using the supergravity language, we can simply perform a T-duality transformation on the small spatial circles. This produces a metric of $N$ coincident D0-branes of Type-IIA string theory, 'smeared' over the torus $\mathbf{T}^{p}$, which now has a local size larger than the string scale:

$$
d s^{2}\left(\widetilde{X}_{\mathrm{bh}}\right)=\frac{h}{\sqrt{H}} d \tau^{2}+\sqrt{H}\left(d \overrightarrow{\tilde{y}}^{2}+\frac{d r^{2}}{h}+r^{2} d \Omega_{8-p}^{2}\right),
$$

where we have defined the dual coordinates on the T-dual torus with identification $\tilde{y} \equiv$ $\tilde{y}+\widetilde{L}$, with $\widetilde{L}=(2 \pi)^{2} \ell_{s}^{2} / L$, and the harmonic functions $H$ and $h$ as in (2.1), (2.14). At $r \sim r_{L}$, this manifold is glued to the previous one (2.13) or (2.16) by a 'neck' of stringy size and $\mathcal{O}(1)$ curvatures. The cross-over temperature at which we go over the T-dual language is determined by $r_{0} \sim r_{L}$ :

$$
T\left(X_{\mathrm{bh}} \leftrightarrow \widetilde{X}_{\mathrm{bh}}\right) \sim T_{\mathrm{Hag}}\left(\frac{\ell_{s}}{L}\right)^{\frac{2(5-p)}{7-p}}\left(g_{s} N\right)^{\frac{1}{p-7}} .
$$

The corresponding T-dual vacuum manifold is obtained by setting $r_{0}=0$ in (5.2). As before, its classical free energy is vacuum-dominated and it is thermodynamically subleading for $p<5$, and dominating for $p \geq 5$.

In fact, at sufficiently low temperatures, the system of 'smeared' black $D 0$-branes, with metric (5.2), is unstable towards localization in the spacetime torus $\mathbf{T}^{p}$, thus breaking the translational isometries. This was described in [25], and related in [26] to the 'fractionalization effect', or $D$-brane wrapping, first pointed out in [27]. The metric is the 'array solution' of periodically identified ten-dimensional $D 0$-branes on a $\mathbf{Z}^{p}$ grid in the $\tilde{y}$ directions $\overrightarrow{\tilde{y}}=\vec{n} \widetilde{L}$ :

$$
d s^{2}\left(X_{\mathrm{D} 0}\right)=\frac{h_{0}}{\sqrt{H_{0}}} d \tau^{2}+\sqrt{H_{0}}\left(\frac{d \rho^{2}}{h_{0}}+\rho^{2} d \Omega_{8}^{2}\right),
$$

with $\rho$ a ten-dimensional radial coordinate. Taking the near-horizon limit, we have $H_{0} \sim$ $\sum_{\vec{n}} b_{0}^{7} /|\vec{\rho}-\vec{n} \widetilde{L}|^{7}, h_{0} \sim 1-\sum_{\vec{n}} \rho_{0}^{7} /|\vec{\rho}-\vec{n} \widetilde{L}|^{7}$, and we can actually neglect the effect of the images. The charge radius is $b_{0} \equiv \ell_{s}\left(\tilde{g}_{s} N\right)^{1 / 7}$, with the T-dual asymptotic string coupling 
given by $\tilde{g}_{s}=g_{s}\left(\ell_{s} / L\right)^{p}$, so that the charge radii of both manifolds satisfy the matching $b_{0}^{7} \sim \widetilde{L}^{p} b^{7-p}$.

The thermodynamical balance between (5.2) and (5.4) follows slightly different patterns in the microcanonical and the canonical ensembles. In the microcanonical ensemble (see for example [26],) we compare the entropies, at a fixed value of the mass. Matching the ADM mass above extremality for both (5.2) and (5.4), we have

$$
E\left(\widetilde{X}_{\mathrm{bh}}\right) \sim \frac{\widetilde{L}^{p}}{\widetilde{G}} r_{0}^{7-p} \sim E\left(X_{\mathrm{D} 0}\right) \sim \frac{1}{\widetilde{G}} \rho_{0}^{7},
$$

where $\widetilde{G}$ is the ten-dimensional T-dual Newton constant (notice that the combination $\widetilde{L}^{p} / \widetilde{G}$ is T-duality invariant.) From (5.5) we obtain the matching $\rho_{0}^{7} \sim \widetilde{L}^{p} r_{0}^{7-p}$. comparing now the entropies one finds that $\widetilde{X}_{\mathrm{bh}}$ dominates for $r_{0} \gg \widetilde{L}$, while $X_{\mathrm{D} 0}$ does so for $r_{0} \ll \widetilde{L}$.

On the other hand, in the canonical ensemble, it is the temperature of both manifolds, (2.19), that has to be matched. One then finds a relation between the horizons of the form

$$
\rho_{0}^{5} \sim \widetilde{L}^{p} r_{0}^{5-p}
$$

Now the ratio of the free energies scales

$$
\frac{I\left(\widetilde{X}_{\mathrm{bh}}\right)}{I\left(X_{\mathrm{D} 0}\right)} \sim(5-p) \frac{\beta \widetilde{L}^{p} r_{0}^{7-p}}{\beta \rho_{0}^{7}} \sim(5-p)\left(\frac{r_{0}}{\widetilde{L}}\right)^{\frac{2 p}{5}} .
$$

Therefore, as long as $p<5$, the critical line for the thermodynamical balance between $\widetilde{X}_{\mathrm{bh}}$ and $X_{\mathrm{D} 0}$ is again $r_{0} \sim \widetilde{L} \sim \ell_{s}^{2} / L$. In terms of the gauge theory termperature, the transition locus defines the curve

$$
T\left(\widetilde{X}_{\mathrm{bh}} \leftrightarrow X_{\mathrm{D} 0}\right) \sim T_{\mathrm{Hag}}\left(\frac{\ell_{s}}{L}\right)^{\frac{5-p}{2}}\left(g_{s} N\right)^{-\frac{1}{2}},
$$

with $X_{\mathrm{D} 0}$, the localized solution, dominating the lower temperature, weaker coupling, regime. Both lines intersect a a value of the coupling $g_{s} N \sim\left(\ell_{s} / L\right)^{3-p}$ and $T \sim 1 / L$. This is very satisfying, since this is the temperature for the onset of finite-size effects in the weak coupling description of the gauge theory.

The transition between $\widetilde{X}_{\mathrm{bh}}$ and $X_{\mathrm{D} 0}$ can be established as a genuine large $N$, firstorder phase transition of the gauge theory, similar to the Gross-Witten phase transition [28] (see also [29], [30].) It is the analogue, for toroidal geometry, of the 'flop' described in [6], [7] for CFTs in spheres. 
Again, for $p \geq 5$, the classical free energy of $\widetilde{X}_{\mathrm{bh}}$ becomes positive because of the factor of $5-p$ in front, as in (5.7). As a result, the solution of smeared D0-branes is suppressed in the canonical ensemble, and we only have $X_{\mathrm{D} 0}$ dominance below the line (5.8).

A non-perturbative threshold develops when the T-dual dilaton in the metric (5.2), or the $D$ 0-brane dilaton in (5.4), becomes large at still lower values of the radial coordinate. At this point we must turn to an eleven-dimensional description, along the lines of [13], where new 'localization' phase transitions can occur, as in [31], [32]. In this paper, we are neglecting such non-perturbative thresholds as $N$ is taken hierarchically larger than any other dimensionless quantity in the problem.

The relation of the critical radius $r_{L}$ to finite-size effects can be established heuristically through the calculation of spatial Wilson loops, along the lines of [33]. Consider a Wilson loop defined at the upper boundary of the throat with length $\ell$. In the semi-classical approximation we must evaluate the area of the minimal surface in $X$ with boundary the Wilson loop of length $\ell$. An area-law contribution arises whenever the minimal world-sheet 'saturates' at some radius, signaling the end of the manifold $X$. This happens at the horizon for $X_{\mathrm{bh}}$, [7], [34], where $r_{\mathrm{sat}} \sim r_{0}$. In general, the area-law contribution to the Wilson loop takes the form

$$
\left\langle W_{\ell}\right\rangle_{X} \sim e^{-A_{\text {sat }} / \alpha^{\prime}},
$$

where the saturated area is given by the red-shifted area of the loop in the $\vec{y}$-space:

$$
\frac{A_{\mathrm{sat}}}{\alpha^{\prime}} \sim \frac{1}{\alpha^{\prime}}\left(\frac{r_{\mathrm{sat}}}{b_{r}\left(r_{\mathrm{sat}}\right)}\right)^{2} \ell^{2}=\frac{\left(g_{s} N\right)^{\frac{2}{7-p}}}{b^{2}}\left(\frac{r_{\mathrm{sat}}}{b}\right)^{\frac{7-p}{2}} \ell^{2} .
$$

The square root of the string tension, or effective mass gap, is related to the saturation radius by

$$
M_{\text {gap }} \sim \frac{\left(g_{s} N\right)^{\frac{1}{7-p}}}{b} \cdot\left(\frac{r_{\text {sat }}}{b}\right)^{\frac{7-p}{4}} .
$$

Since the spatial sections of the manifold $X_{\mathrm{vac}}$ ( or $X_{\mathrm{bh}}$ for $r_{0} \ll r_{L}$,) are 'pinched' to stringy size at $r \sim r_{L}$, it seems natural to assume that, for the purposes of the semiclassical approximation to the calculation of Wilson loops, the neck at $r_{L}$ represents a cut-off where the minimal area world-sheet can saturate. In other words, we can assume that the manifold $X$ 'ends' at $r \sim r_{L}$, so that we may take $r_{\text {sat }} \sim r_{L}$, leading to an induced gap

$$
M_{\text {gap }} \sim \frac{1}{L}
$$


so that the saturated Wilson loop scales

$$
\left\langle W_{\ell}\right\rangle \sim e^{-C \ell^{2} / L^{2}}
$$

that is, it has the interpretation of the Wilson loop hitting the walls of the spatial box.

\subsection{Thermal Winding Modes and Hagedorn Effects}

We can generalize the discussion of finite-size effects to the temporal winding modes. In this case we have Hagedorn behaviour at $r \sim r_{\beta}$ and we cannot exhibit a geometric picture of the inner region at $r \ll r_{\beta}$, due to the lack of control over duality transformations in the absence of supersymmetry. However, since the world-sheet physics is similar (infrared divergences due to light winding modes), it seems natural to postulate a similar geometric picture in terms of a 'pinching effect' at $r \sim r_{\beta}$. This leads to a cut-off version of $X_{\text {vac }}$ at the Hagedorn radius $r_{\beta}$ which we call $X_{\text {Hag. }}$. In fact, assuming that spatial Wilson loops saturate at $r_{\text {sat }} \sim r_{\beta}$, we get an associated gap from formula (5.11):

$$
M_{\mathrm{mag}} \sim \frac{1}{\beta}
$$

This has the interpretation of the 'magnetic mass gap' of the effective $p$-dimensional theory arising in the high temperature limit. In this case, we see that the gap induced by $X_{\mathrm{Hag}}$ is no other than the standard scale of Matsubara modes. This gap is to be contrasted to the black-hole induced mass gap, with saturation at the horizon $r_{\text {sat }} \sim r_{0}$, which scales like [35], 36]:

$$
M\left(X_{\mathrm{bh}}\right)_{\mathrm{mag}} \sim \frac{\left(g_{s} N\right)^{\frac{1}{7-p}}}{b}\left(\frac{b}{\beta}\right)^{\frac{7-p}{2(5-p)}}=\left[\left(g_{\mathrm{YM}}^{2} N\right) T^{7-p}\right]^{\frac{1}{2(5-p)}}
$$

In fact, in the supergravity regime $g_{s} N \ll 1$, we tend to have $M\left(X_{\mathrm{bh}}\right) \gg T$. However, before concluding that $X_{\text {Hag }}$ dominates over $X_{\mathrm{bh}}$ in the contribution to Wilson loop expectation values, we have to determine the $\mathcal{O}\left(e^{-N^{2}}\right)$ prefactors in the expectation value (5.9), i.e. the relative free energies of $X_{\mathrm{bh}}$ and $X_{\mathrm{Hag}}$.

In order to estimate the free energy of $X_{\mathrm{Hag}}$, we shall adopt a 'phenomenological' attitude, and assume some standard qualitative facts about the Hagedorn behaviour of thermal strings. In a world-sheet picture the Hagedorn transition can be described [37] as a BKT phase transition on the world-sheet [38]. The world-sheet vortices are the vertex 
operators for winding modes and their condensation means that some of the winding modes become tachyonic. Due to this world-sheet defects, a genus-zero free energy is generated according to [39]. Translated to the gauge theory language, this means that a planar term is induced for the free energy of $X_{\mathrm{vac}}$ or, in other words, a classical entropy

Thus, we assume that, for thermodynamical purposes, strong-coupling effects associated with massless winding modes effectively modify the $X_{\mathrm{vac}}$ manifold, capping it at distances of order $r_{\beta}$, and generating a 'stretched horizon' 5 The free energy of the resulting capped manifold $X_{\mathrm{Hag}}$ will be calculated as

$$
I\left(X_{\mathrm{Hag}}\right)=\beta M\left(X_{\mathrm{Hag}}\right)-S\left(X_{\mathrm{Hag}}\right)
$$

We approximate the entropy by the area of the stretched horizon,

$$
S\left(X_{\mathrm{Hag}}\right) \sim \frac{A_{\mathrm{Hag}}}{4 G_{d}}=\frac{\operatorname{Vol}\left(\mathbf{S}^{8-p}\right) L^{p}}{4 G} r_{\beta}^{8-p} \sqrt{H\left(r_{\beta}\right)} \sim \frac{\operatorname{Vol}\left(\mathbf{S}^{8-p}\right) L^{p}}{4 G} b^{\frac{7-p}{2}} r_{\beta}^{\frac{9-p}{2}}
$$

where we have used $r_{\beta} \ll b$. The energy of the stretched horizon can be estimated, on the basis of dimensional analysis, and translational and rotational symmetry, to take the form of (3.8), with $r_{0}$ replaced by $r_{\beta}$ :

$$
M\left(X_{\mathrm{Hag}}\right)-M_{E D p} \sim \frac{\operatorname{Vol}\left(\mathbf{S}^{8-p}\right) L^{p}}{16 \pi G} r_{\beta}^{7-p},
$$

up to constants of $\mathcal{O}(1)$. Solving for $r_{\beta}$ as a function of the temperature, we find that the contribution of the internal energy (5.18) to the canonical free energy (5.16) tends to make the specific heat negative. Let us assume for the time being that the entropy of the stretched horizon largely dominates compared to its internal energy:

$$
S\left(X_{\mathrm{Hag}}\right) \gg \beta\left(M\left(X_{\mathrm{Hag}}\right)-M_{E D p}\right)
$$

Comparing the two terms, using (2.12), this is satisfied provided $\beta \gg \ell_{s}\left(g_{s} N\right)^{\frac{1}{p-3}}$ for $p<3$, and $\beta \ll \ell_{s}\left(g_{s} N\right)^{\frac{1}{p-3}}$ for $p>3$. In the conformal case, (5.19) is met when $g_{s} N \gg 1$. These assumptions will be justified later. Under these circumstances, the resulting free energy takes the form

$$
I\left(X_{\mathrm{Hag}}\right)=-N^{2} C^{\prime}\left(g_{s} N\right)^{\frac{2(5-p)(p-6)}{(7-p)^{2}}} L^{p} b^{\gamma^{\prime}-p} \beta^{-\gamma^{\prime}}+\beta E_{\mathrm{vac}}
$$

\footnotetext{
4 Other discussions appear in [40].

5 Locating the stretched horizon at the point where the local temperature is the Hagedorn temperature is natural also in other contexts, see [41].
} 
with $C^{\prime} \sim \mathcal{O}(1)$ and positive, and

$$
\gamma^{\prime}=\frac{2(9-p)}{7-p}
$$

This critical exponent is harder than $\gamma_{\text {free }}=p$, and also harder than (3.17) for $p<3$. It equals the conformal one for $p=3$, and it is softer than either (3.17) or $\gamma_{\text {free }}$ for $3<p<6$. We have $\gamma^{\prime}=\gamma_{\text {free }}$ at $p=6$. With these values of $\gamma^{\prime}$, given the positivity of $C^{\prime}$, the manifold $X_{\text {Hag }}$ has positive specific heat up to $p=7$, where the analysis breaks down.

A very interesting feature of $(5.20)$ is that, just as in (4.12), the dependence on the closed string scale $\sqrt{\alpha^{\prime}}$, and the effective D-brane coupling $g_{s} N$, combine in such a way that the physical free energy only depends on the Yang-Mills coupling,

$$
I\left(X_{\mathrm{Hag}}\right)=\beta E_{\mathrm{vac}}-N^{2} B^{\prime}\left(g_{\mathrm{YM}}^{2} N\right)^{\frac{p-6}{7-p}} L^{p} \beta^{-\gamma^{\prime}} .
$$

\subsection{Hagedorn Censorship}

In order to determine whether the vacuum manifolds capped at the Hagedorn stretched horizon, $X_{\mathrm{Hag}}$, can dominate over the smooth $X_{\mathrm{bh}}$ manifolds in the canonical ensemble, we compare the actions evaluated above. We find for the difference,

$$
\beta F(\beta)_{\mathrm{planar}}=I\left(X_{\mathrm{bh}}\right)_{c \ell}-I\left(X_{\mathrm{Hag}}\right)_{c \ell}=-\frac{\mathrm{Vol}\left(\mathbf{S}^{8-p}\right) L^{p}}{4 G} b^{\frac{7-p}{2}}\left(\frac{5-p}{2(7-p)} r_{0}^{\frac{9-p}{2}}-C_{h} r_{\beta}^{\frac{9-p}{2}}\right),
$$

with $C_{h}$ a positive constant of order one, which summarizes our ignorance in the treatment of the stretched horizon at $r_{\beta}$. Thus, we see that the criterion for $X_{\mathrm{bh}}$ dominance in the large $N$ limit is $r_{0} \gg r_{\beta}$, provided $p<5$. For $p \geq 5$, the difference in $(5.22)$ is positive and $X_{\text {Hag }}$ dominates the canonical ensemble to leading order. At lower temperatures, we must compare the T-dual manifolds $\widetilde{X}_{\mathrm{bh}}$ and $X_{\mathrm{D} 0}$, with the corresponding vacuum manifolds capped at the Hagedorn radius. The Hagedorn radius and free energy of $\widetilde{X}_{\text {Hag }}$ are the same as $X_{\text {Hag }}$ in $(5.22)$, since T-duality of the metric in the spatial torus $\mathbf{T}^{p}$ has no effect on the thermal direction. On the other hand, for the array solution, the Hagedorn radius is $r_{\beta 0} \sim b_{0}\left(\ell_{s} T\right)^{4 / 7}$, and the free energy scales like $I\left(X_{\operatorname{Hag} 0}\right) \sim b_{0}^{7 / 2} \rho_{0}^{9 / 2} / \widetilde{G}$. 


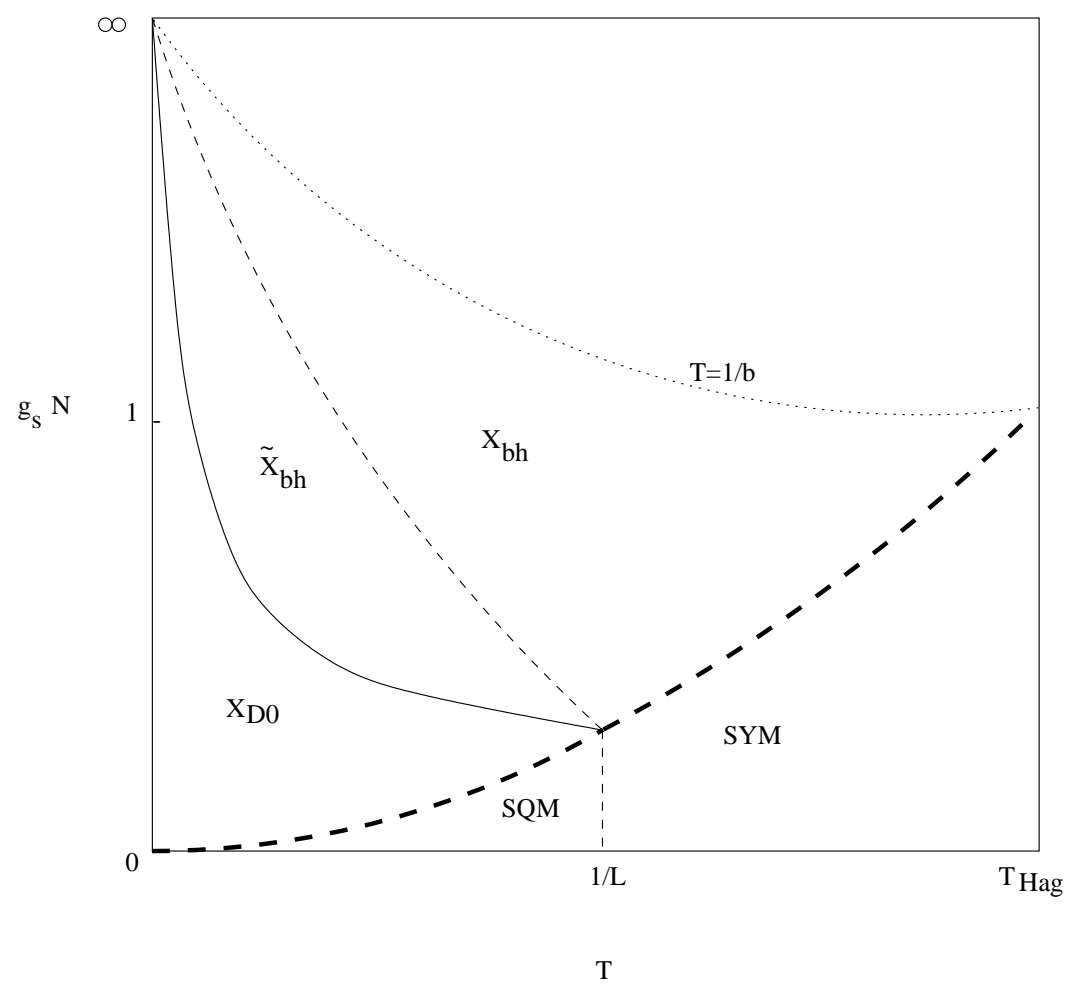

Fig. 4: Phase diagram for $p<3$

We now study the pattern of large $N$ 'phase transitions' between the different descriptions of the system. In those cases where large $\alpha^{\prime}$ corrections are involved, either locally (transitions to $X_{\mathrm{Hag}}$ or the $X_{\mathrm{bh}} \leftrightarrow \widetilde{X}_{\mathrm{bh}}$ T-duality,) or globally (transitions to the SYM regime at $r \sim r_{c}$,) we cannot decide, on the basis of our low-energy supergravity analysis, whether we have real large $N$ phase transitions, as advocated recently in [42], or only smooth cross-over regions. It was argued in [36] that the transition between the $X_{\mathrm{bh}}$ manifold and the SYM description involves no $\mathcal{O}\left(N^{2}\right)$ jump in the entropy, unlike the real large $N$ phase transition between $X_{\mathrm{bh}}$ and $X_{\mathrm{vac}}$ described in [6], [7]. However, the 'flop' between $\widetilde{X}_{\mathrm{bh}}$ and $X_{\mathrm{D} 0}$, described in subsection 5.1, is a large $N$ first-order phase transition with exact matching of the entropies.

The supergravity regime is defined by the requirement that the interesting features of $X_{\mathrm{bh}}$ or $X_{\mathrm{Hag}}$ be clearly separated both from the onset of finite-size effects, and from large curvature corrections in the bulk. For $p \leq 3$, this leads to the conditions $r_{0}, r_{\beta} \ll r_{c}$, with $r_{c}=\infty$ for $p=3$. For $p>3$, we must require $r_{0}, r_{\beta} \gg r_{c}$. In addition, we exclude from this regimes the regions of parameters where $r_{0}, r_{\beta} \sim r_{L}$. 


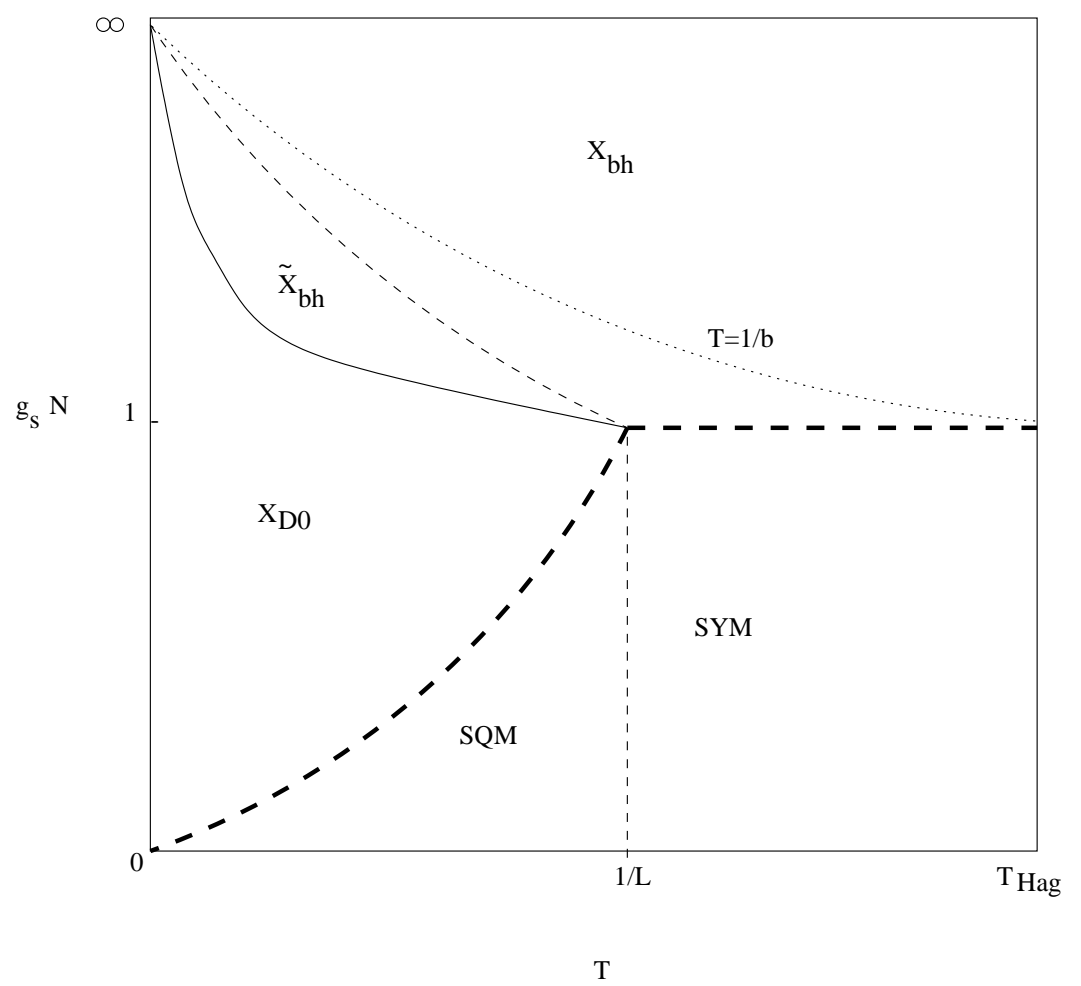

Fig. 5: Phase diagram for $p=3$

One can find the phase diagram by locating the cross-over regimes between different descriptions, when they indeed dominate. The cross-over between the SYM and the black hole $X_{\mathrm{bh}}$ descriptions is estimated by the condition $r_{0} \sim r_{c}$, leading to the following curve in the $\left(T, g_{s} N\right)$ plane:

$$
T\left(\mathrm{SYM} \leftrightarrow X_{\mathrm{bh}}\right) \sim T_{\mathrm{Hag}}\left(g_{s} N\right)^{\frac{1}{3-p}}
$$

In fact, the same curve governs the transition between other, perhaps subleading, descriptions, i.e. as a function of $g_{s} N$ we have

$$
T\left(\mathrm{SYM} \leftrightarrow X_{\mathrm{bh}}\right) \sim T\left(\mathrm{SYM} \leftrightarrow X_{\mathrm{Hag}}\right) \sim T\left(X_{\mathrm{bh}} \leftrightarrow X_{\mathrm{Hag}}\right)
$$

This is related to the fact that the radii $r_{c}, r_{\beta}, r_{0}$ become equal to each other at the same temperature.

The transition curves due to finite-size effects are different. In the SYM regime, we have the obvious temperature scale

$$
T(\mathrm{SYM})_{\mathrm{f} . \operatorname{size}} \sim \frac{1}{L}
$$


the same that one finds for the $X_{\text {Hag }}$ description, determined by $r_{\beta} \sim r_{L}$, i.e.

$$
T\left(X_{\mathrm{Hag}} \leftrightarrow \tilde{X}_{\mathrm{Hag}}\right) \sim \frac{1}{L} .
$$

On the other hand, for the black-hole manifold, the transition to the T-dual description in terms of the smeared $D 0$-branes solution occurs along the curve $r_{0} \sim r_{L}$, or

$$
T\left(X_{\mathrm{bh}} \leftrightarrow \widetilde{X}_{\mathrm{bh}}\right) \sim T_{\mathrm{Hag}}\left(\frac{\ell_{s}}{L}\right)^{\frac{2(5-p)}{7-p}}\left(g_{s} N\right)^{\frac{1}{p-7}} .
$$

Finaly, the transition due to the localization effect between $\widetilde{X}_{\mathrm{bh}}$ and $X_{\mathrm{D} 0}$ lies on the curve (5.8):

$$
T\left(\widetilde{X}_{\mathrm{bh}} \leftrightarrow X_{\mathrm{D} 0}\right) \sim T_{\mathrm{Hag}}\left(\frac{\ell_{s}}{L}\right)^{\frac{5-p}{2}}\left(g_{s} N\right)^{-\frac{1}{2}} .
$$

Since $X_{\mathrm{D} 0}$ dominates the supergravity regime in the low temperature, weak coupling regime, the transition to the SYM description at temperatures below (5.25) is determined by the curvature threshold of $X_{\mathrm{D} 0}$. The resulting cross-over can be obtained from (5.23) by putting $p=0$, and substituting $g_{s}$ by its T-dual:

$$
T\left(\mathrm{SYM} \leftrightarrow X_{\mathrm{D} 0}\right) \sim T_{\mathrm{Hag}}\left(\tilde{g}_{s} N\right)^{\frac{1}{3}} \sim T_{\mathrm{Hag}}\left(\frac{\ell_{s}}{L}\right)^{\frac{p}{3-p}}\left(g_{s} N\right)^{\frac{1}{3}} .
$$

The localized D0-brane metric actually matches to the quantum mechanics of the zero modes of SYM on $\mathbf{T}^{p}$, at energies below the gap 1/L, which we denote SQM in the pictures. According to [26], the entropically subleading 'wrapped' system, which behaves extensively in $p$ dimensions down to very low temperatures, matches to the (also subleading) 'smeared' metric $\widetilde{X}_{\mathrm{bh}}$. It is tempting to conjecture that the SYM theory on $\mathbf{T}^{p}$ with twisted boundary conditions (no 'toron' zero-modes) would have a behaviour more similar to the $\mathbf{S}^{3}$ case studied in [6], [7], i.e. without a phase dominated by localized D0-branes.

With this information we can determine the 'phase diagrams' as in figures 4,5,6 and 7 below. In the figures, we have considered only 'field theoretic temperatures' in the brane theory, $T$, up to the string scale $T_{\text {Hag }} \sim \ell_{s}^{-1}$, postponing to section 7 the study of some aspects of the physics at boundary temperatures of the order of the Hagedorn temperature. We also plot, for future convenience, the curve $T=b^{-1}$, representing the maximal temperature of the complete black-brane manifold $B D p$. The large $N$ phase transitions due to finite-size effects, (5.28), appear as continuous lines in the diagrams, whereas the less understood transitions appear in dashed lines: thick lines for the crossover between the black-hole and SYM descriptions, the curves (5.23), (5.29), and thin lines for the T-duality transition (5.27). Notice that all curves meet at the same point in the $\left(g_{s} N, T\right)$ plane, given by $T=1 / L$ and $g_{s} N=\left(\ell_{s} / L\right)^{3-p}$. 


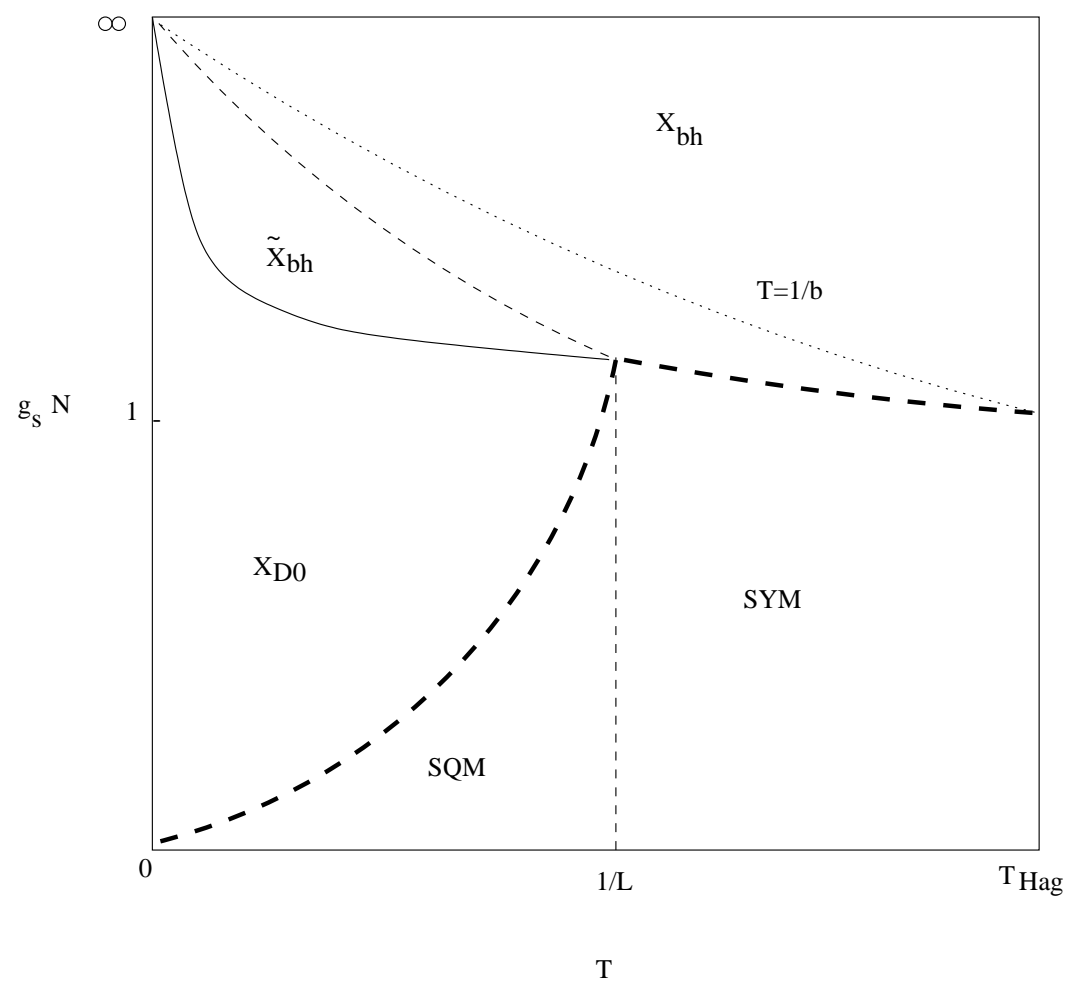

Fig. 6: Phase diagram for $p=4$

The salient features of the analysis are the following. First, the conditions of validity of (5.19) are exactly equivalent to the condition that we are not in the SYM regime, according to (5.24). Therefore, (5.19) holds in the region where $X_{\text {Hag }}$ could contribute. However, one finds some hierarchies between the radii: $r_{\beta} \ll r_{0} \ll r_{c}$ for the supergravity regime in $p \leq 3$, and $r_{c} \ll r_{\beta} \ll r_{0}$ for the supergravity regime in $p=4$, with $r_{\beta} \sim r_{0}$ only at the cross-over to the SYM description, i.e. $r_{\beta} \sim r_{0} \sim r_{c}$ at the same temperature given by (5.23) and (5.24). The result is identical for the $X_{\mathrm{D} 0}$ horizon, at $\rho=\rho_{0}$, versus the Hagedorn radius on the array solution, $r_{\beta 0} \sim b_{0}\left(\ell_{s} T\right)^{4 / 7}$. It is also interesting to compare the Hagedorn radii of the smeared and localized $D 0$-brane solutions. One finds that, at very low temperatures, $r_{\beta} \ll r_{\beta 0}$, and the cross-over between $\widetilde{X}_{\mathrm{Hag}}$ and $X_{\mathrm{Hag} 0}$ takes place along the curve

$$
T\left(\widetilde{X}_{\mathrm{Hag}} \leftrightarrow X_{\mathrm{Hag} 0}\right) \sim T_{\mathrm{Hag}}\left(\frac{\ell_{s}}{L}\right)^{\frac{7-p}{4}}\left(g_{s} N\right)^{-\frac{1}{4}} .
$$

This curve also meets all of the others at the $T \sim 1 / L$ common point.

Thus, by comparing the canonical free energies, one concludes that $X_{\mathrm{Hag}}$ never dominates the canonical ensemble as long as $p<5$. Thus we have a true thermodynamical 'Hagedorn censorship' for these values of $p$. 


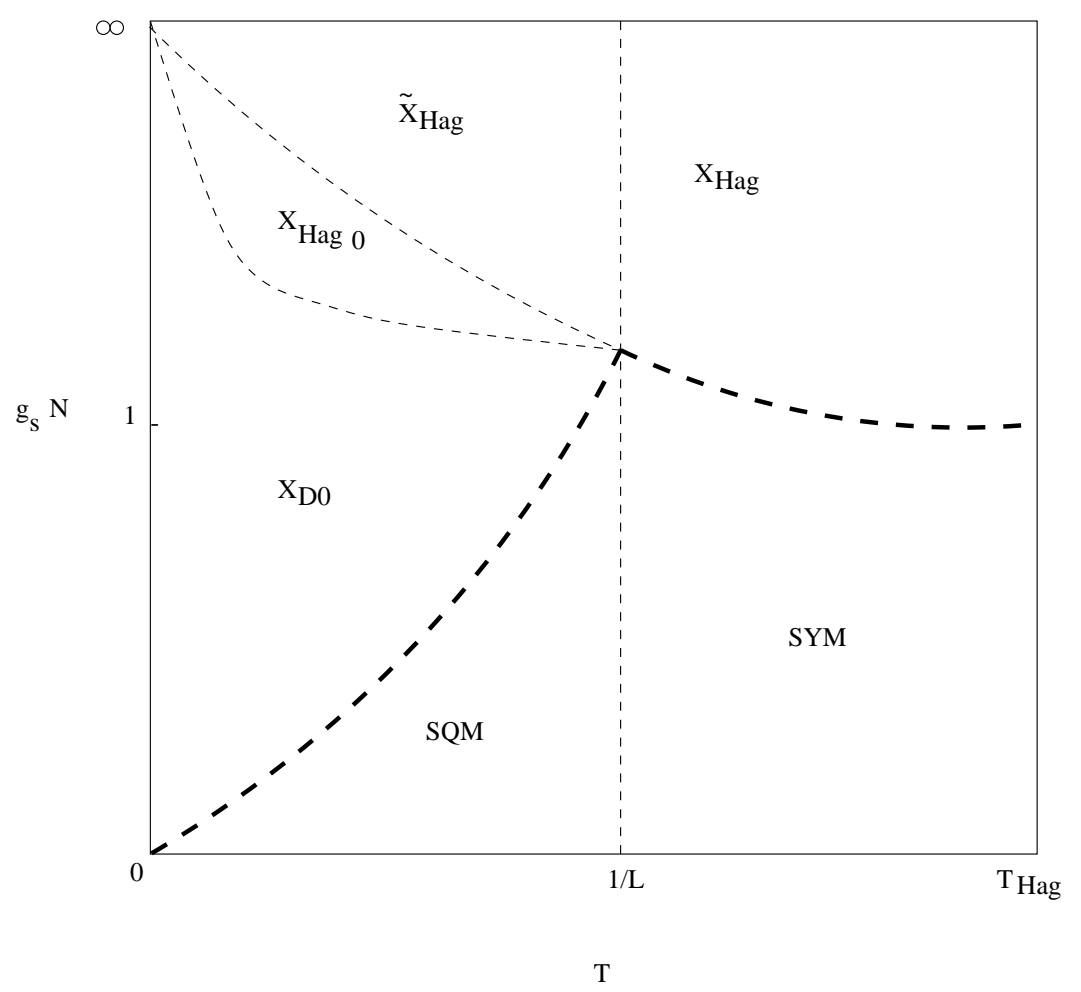

Fig. 7: Phase diagram for $p=5,6$

On the other hand, for $p=5,6$, the black-hole manifold $X_{\mathrm{bh}}$ has positive free energy and is suppressed in the canonical ensemble at high temperature. Then $X_{\text {Hag }}$ dominates the supergravity regime. In particular, for $p=6$ the resulting scaling of the planar free energy is the same as in a seven-dimensional free field theory.

In the low temperature region, there is a cross-over at $T \sim 1 / L$ to $\widetilde{X}_{\text {Hag. }}$. At lower temperatures or couplings, (5.30), $X_{\text {Hag0 }}$ becomes thermodynamically more favorable, and finally, at still lower temperatures (5.28), the smooth manifold with positive specific heat $X_{\mathrm{D} 0}$, with the array of localized $D 0$-branes, dominates and makes contact with the SQM description.

Therefore, we do find thermodynamical domination of manifolds with Hagedorn cutoffs for $p=5,6$. However, we shall find in the next section that, as soon as $p \geq 5$, one cannot make sense of the $1 / N$ corrections in terms of a gauge theory in $p+1$ dimensions.

The Hagedorn censorship can be turned into a Hagedorn exposure if we switch to the regime $g_{s} N \ll 1$. In this case $r_{\beta}$ tends to be much larger than $r_{0}$. On the other hand, the whole approach of comparing smooth manifolds breaks down, as the $\alpha^{\prime}$ corrections become 
of order one everywhere. This is indeed the regime where D-brane perturbation theory is a good approximation.

If we nevertheless pursue the geometric analysis, in a formal fashion, beyond its range of applicability, one can see that $X_{\mathrm{Hag}}$ formally dominates $\left(r_{\beta}>r_{0}\right)$ for sufficiently high temperatures $\ell_{s} \ll \beta \ll b / r_{c}$, for $p<3$. For $p \geq 3$ and $g_{s} N \ll 1$, we have $X_{\mathrm{Hag}}$ dominance for all temperatures below the string scale. In fact, the Hagedorn cut-off manifolds overestimate the entropy with respect to the SYM answer, i.e. if we evaluate the ratio $\left|I\left(X_{\mathrm{Hag}}\right) / I(\mathrm{SYM})\right|$ in the SYM regime, it is strictly larger than one for all $p<6$, and equal to one, in order of magnitude, precisely for $p=6$. In any case, the resulting hightemperature string tension in the effective $p$-dimensional theory, does not agree with the perturbative determination, and therefore it is hard to determine the relevance of this geometric picture at $g_{s} N \ll 1$.

\section{Holography Criterion at One Loop}

As pointed out in [10], a necessary condition for holography to hold in an operational sense is that, by heating up the gauge theory, we do not uncover the full ten- or elevendimensional structure of the supergravity description. This can be tested by estimating the one-loop free energy in the supergravity side, which should be interpreted as the leading $1 / N^{2}$ correction on the gauge theory side.

In a space with locally varying Kaluza-Klein thresholds, the number of effective dimensions is given by the number of 'circles' with proper radius larger than the local inverse temperature. In a region $X\left(d_{\text {eff }}\right)$ where this number of effective dimensions is fixed, $d_{\text {eff }}=\operatorname{dim}\left(X\left(d_{\text {eff }}\right)\right)$, we can estimate the one-loop free energy by a red-shift [8], or WKB formula 43 :

$$
\beta F_{\mathrm{WKB}} \sim-\int_{X\left(d_{\mathrm{eff}}\right)} d \mathrm{Vol}\left(\frac{1}{\beta \sqrt{g_{00}}}\right)^{d_{\mathrm{eff}}}+\beta E_{\mathrm{vac}} .
$$

In other words, the one-loop free energy in curved space is approximately extensive (in the standard sense of linearity) in the so-called optical volume [44], i.e. the volume measured in the optical metrid 6 ,

$$
\widetilde{g}_{\mu \nu}=\frac{g_{\mu \nu}}{g_{00}}
$$

6 Notice that the optical metric $\widetilde{g}_{\mu \nu} \equiv g_{\mu \nu} / g_{00}$ is invariant under conformal rescalings of $g_{\mu \nu}$. This means that it is the same for the string or Einstein frames. In fact, it is the Einstein-frame metric the relevant one for one-loop computations in supergravity, because the dilaton has a negative kinetic energy in the string frame metric. 
For a manifold with several thresholds with different effective dimensions $d_{i}$, we have

$$
\beta F(\beta)_{1-\mathrm{loop}} \sim-\sum_{X_{d_{i}}} A_{i} \beta^{-d_{i}} \widetilde{\operatorname{Vol}}\left(X_{d_{i}}\right)+\beta E_{\mathrm{vac}},
$$

where the constants $A_{i}$ are related to the number of degrees of freedom. In the present case, $A_{i} \sim \mathcal{O}(1)$ in the large $N$ limit. Notice that the real temperature scaling of the contribution from a given region $X_{d_{i}}$ could differ from $T^{d_{i}}$, because of the temperature dependence of the optical volume, hidden in the definition of the limits of the region $X_{d_{i}}$ itself.

The expression (6.3) was analyzed in [10] for the conformal cases of the form $X_{\mathrm{vac}}=$ $A d S_{d+1} \times M_{D-d-1}$, with $M_{D-d-1}$ a compact manifold of constant curvature and radius $b$. Examples include the CFT on the $D 3$-branes world-volume for $D=10$, and the $M 2$ - and $M 5$-branes world-volume CFTs for $D=11$. In all these cases, the black-hole manifold $X_{\mathrm{bh}}$ takes the form of an AdS Schwarzschild black-hole extended over $M_{D-d-1}$ and with horizon radius $r_{0} \sim b^{2} T_{0}$, with $T_{0}$ the local temperature at the centre of the $X_{\text {vac }}$ space. The condition of decoupling of the Kaluza-Klein threshold of the compact manifold $M_{D-d-1}$ leads to an asymptotic region with $d+1$ effective dimensions, and a 'core' region with $D$ effective dimensions. The cross-over is at $r_{\mathrm{dec}} \sim r_{0}$, so that $X_{\mathrm{bh}}$ is completely $d+$ 1 dimensional for thermal purposes, while $X_{\text {vac }}$ has a 'core' for $r<b^{2} T_{0}$ where it is effectively $D$-dimensional. Computing now the optical volume of the different regions and plugging it in the general expression (6.3), one finds that $X_{\text {vac }}$ is non-holographic, with a $D$-dimensional scaling behaviour $\sim T^{D-1}$, coming from the core. The asymptotic region of both $X_{\mathrm{vac}}$ and $X_{\mathrm{bh}}$ yields always a holographic contribution to the free energy of the form $\sim T^{d-1}$. Hence, one concludes that $X_{\mathrm{bh}}$ dominance guarantees holographic behaviour as characterized by the power dependence of the $1 / N^{2}$ corrections to the free energy.

We generalize now the discussion to the the more general non-conformal cases treated in this paper. In fact, we can carry out the analysis for all the branes of low-energy superstring theory at once, using the general solutions of [15],

$$
d s^{2}=\frac{1}{H^{\delta(a+1)}}\left(h d \tau^{2}+d \vec{y}^{2}\right)+\frac{1}{H^{\delta(a-1)}}\left(\frac{d r^{2}}{h}+r^{2} d \Omega_{8-p}^{2}\right),
$$

where $a=0$ for $D p$-branes, $a=-1$ for NS fivebranes and $a=1$ for the fundamental string solution. The constant $\delta$ is

$$
\delta=\frac{1}{2 a^{2}-(3-p) a+2} .
$$


Notice that $\delta=1 / 2$ for all $D p$-branes as well as the NS five and one-branes, i.e. for all BPS branes of the ten-dimensional type II supergravity or truncations thereof.

The decoupling of the angular sphere $\mathbf{S}^{8-p}$, with a position dependent radius given by

$$
R_{\text {local }}=\frac{r}{(\sqrt{H})^{\delta(a-1)}},
$$

depends on the ratio between this radius and the local inverse temperature

$$
\beta_{\text {local }}=\frac{\beta \sqrt{h}}{(\sqrt{H})^{\delta(a+1)}} .
$$

The ratio scales in the asymptotic, large $r$ region as

$$
\frac{\beta \sqrt{h}}{r} H^{-\delta} \sim \frac{\beta}{b^{(7-p) \delta} r^{1-(7-p) \delta}} .
$$

Therefore, if $1-\delta(7-p)=-(5-p) / 2<0$, the angular sphere $\mathbf{S}^{8-p}$ decouples up the throat. In this case $(p<5)$, the effective holographic manifold for thermal purposes is $X_{p+2}$, obtained from the full ten-dimensional manifold $X$, by dropping the angles, i.e. we remain with the $(\tau, \vec{y}, r)$ components of the metric, or $A d S_{p+2}$ with a variable radius $R_{\text {local }}$.

If $1-\delta(7-p)=0$, or $p=5$, the decoupling depends on the ratio $\beta / b$. For $\beta \gg b$, the angular sphere decouples, but it must be kept in the opposite, high temperature regime $\beta \ll b$.

On the other hand, if $1-\delta(7-p)>0$, or $p>5$, then the angular radius is larger than the local inverse temperature up the throat, and these extra dimensions do not decouple from the thermal partition function.

In all cases, except the marginal one at $p=5$, the radial cut-off defining the asymptotic region can be taken as the radius at which $\beta_{\text {local }} \sim R_{\text {local }}$, or

$$
r_{\mathrm{dec}}^{1-\delta(7-p)} \sim \frac{\beta}{b^{(7-p) \delta}} .
$$

The full optical metric in the asymptotic region, with $h \sim 1$, is

$$
\widetilde{d}^{2}(X) \rightarrow d \tau^{2}+d \vec{y}^{2}+H^{2 \delta}\left(d r^{2}+r^{2} d \Omega_{8-p}^{2}\right),
$$

with optical volume between two limits given by

$$
\begin{aligned}
{[\widetilde{\operatorname{Vol}}(X)]_{r_{\min }}^{r_{\max }} } & =\beta L^{p} \operatorname{Vol}\left(\mathbf{S}^{8-p}\right) \int H^{\delta(9-p)} r d r \\
& =\beta L^{p} \operatorname{Vol}\left(\mathbf{S}^{8-p}\right) b^{\delta(7-p)(9-p)}\left[\frac{r^{(9-p)(1-\delta(7-p))}}{(9-p)(1-\delta(7-p))}\right]_{r_{\min }}^{r_{\max }} .
\end{aligned}
$$


On the other hand, the optical metric of $X_{p+2}$ is the same as in (6.8), after dropping the angles (the $d \Omega_{8-p}^{2}$ term), and the optical volume

$$
\left[\widetilde{\operatorname{Vol}}\left(X_{p+2}\right)\right]_{r_{\min }}^{r_{\max }}=\beta L^{p} \int_{r_{\min }}^{r_{\max }} H^{\delta} d r \sim \beta L^{p} b^{\delta(7-p)}\left[\frac{r^{1-\delta(7-p)}}{1-\delta(7-p)}\right]_{r_{\min }}^{r_{\max }} .
$$

Notice that, in both cases, the quantity governing the divergence properties of the optical volume at large radius is

$$
1-\delta(7-p)=-\frac{5-p}{2}
$$

It is interesting to notice that the 'holography index' $1-\delta(7-p)$ is also governing the leading, $\mathcal{O}\left(N^{2}\right)$ thermodynamical properties of the black-brane manifold $X_{\mathrm{bh}}$. By applying (2.18) to the general metric (6.4), one finds exactly the same result for the relation between temperature and horizon location. In the $r_{0} \ll b$ regime:

$$
\beta=\frac{4 \pi}{7-p} r_{0}\left[H\left(r_{0}\right)\right]^{\delta} \sim b^{\delta(7-p)} r_{0}^{1-\delta(7-p)} .
$$

Thus, the correlation between the finiteness of the optical volume and the positive specific heat of the classical black-brane solution is a property of all black branes, independently of their RR or NS character. Incidentally, (6.11) shows that $r_{0} \sim r_{\text {dec }}$, with $r_{\text {dec }}$ the cut-off defined in (6.7) signaling the decoupling of the angular sphere. This is a property of the exactly conformal throats [10], which generalizes to other $D p$-branes.

For $p<5$, the $\mathcal{O}\left(N^{2}\right)$ thermodynamics is dominated by $X_{\mathrm{bh}}$ at sufficiently high temperatures. We can then set $r_{\min }=r_{\text {dec }} \sim r_{0}$, and the resulting one-loop partition function picks a contribution from large radius of the form

$$
\beta F(p<5)_{1-\text { loop }} \sim-T^{p+2}\left[\widetilde{\operatorname{Vol}}\left(X_{p+2}\right)\right]_{r_{0}}^{\infty} \sim-(L T)^{p}
$$

Here we have set $r_{\max }=\infty$, since the integral converges there. It is remarkable that we find a fully holographic answer, characteristic of a $p+1$ dimensional free gas.

For the borderline case $p=5$, the planar approximation to the canonical ensemble leads to $X_{\text {Hag }}$ dominance, hence we must set $r_{\min } \sim r_{\beta}$. There is a critical temperature, $T_{\text {dec }} \sim 1 / b$, for the global decoupling throughout the throat of the angular sphere $\mathbf{S}^{3}$, and we obtain

$$
\beta F(p=5)_{1-\mathrm{loop}} \sim-T^{5+k}\left[\widetilde{\operatorname{Vol}}\left(X_{5+k}\right)\right]_{r_{\beta}}^{r_{\max }} \sim-(L T)^{5}\left(\frac{T}{T_{\mathrm{dec}}}\right)^{k-1} \log \left(\frac{g_{s} N T_{\mathrm{dec}}^{3} r_{\max }}{T^{2}}\right),
$$


with $k=2$ for $T \ll T_{\text {dec }}$, and $k=5$ for $T \gg T_{\text {dec }}$. In both cases, we do get a violation of holography from the dependence on the gauge theory ultraviolet cut-off $r_{\max }$. At moderately high temperatures $\left(L^{-1} \ll T \ll T_{\text {dec }}\right)$, we find a seven-dimensional gas scaling, while the full ten-dimensional scaling is uncovered for temperatures above the angular decoupling threshold.

Finally, for $p=6$, we must use the full optical volume of the $X_{10}$ manifold, as the angular sphere does not decouple at sufficiently large radius. We have argued in the previous section that $X_{\text {Hag }}$ dominates the canonical ensemble at high temperature $T \gg 1 / L$. We can also define a 'decoupling temperature' depending on whether the Hagedorn radius is larger or smaller than the angular decoupling radius. This temperature is thus defined by the condition $r_{\beta} \sim r_{\text {dec }}$ and is given by

$$
T_{\mathrm{dec}} \sim \frac{\left(g_{s} N\right)^{2 / 3}}{b} \sim \frac{T_{\mathrm{Hag}}}{\left(g_{s} N\right)^{1 / 3}}
$$

We have two regimes. If $T \ll T_{\text {dec }}$ the WKB estimate is

$$
\beta F(p=6)_{1-\text { loop }} \sim-T^{8}\left[\widetilde{\operatorname{Vol}}\left(X_{8}\right)\right]_{r_{\beta}}^{r_{\mathrm{dec}}}-T^{10}\left[\widetilde{\operatorname{Vol}}\left(X_{10}\right)\right]_{r_{\mathrm{dec}}}^{r_{\max }}
$$

The first term gives a holographic contribution, of the form

$$
-T^{7} L^{6} \sqrt{b}\left(\sqrt{r_{\mathrm{dec}}}-\sqrt{r_{\beta}}\right) \sim-(L T)^{6}\left(1-\left(T / T_{\mathrm{dec}}\right)^{3}\right)
$$

because the term with ten-dimensional scaling $\sim T^{9}$ is small for $T \ll T_{\text {dec }}$, compared to the holographic one, scaling with the law $\sim T^{6}$. On the other hand, the second term in (6.15) leads to unsuppressed ten-dimensional behaviour, again from the dependence on the ultraviolet cut-off $r_{\max }$ :

$$
-T^{9} L^{6} b^{3 / 2}\left(r_{\max }^{3 / 2}-r_{\mathrm{dec}}^{3 / 2}\right) \sim-T^{9} L^{6}\left(b r_{\max }\right)^{3 / 2}+(L T)^{6}
$$

In the high temperature regime $T \gg T_{\text {dec }}$ we have

$$
\beta F(p=6)_{1-\mathrm{loop}} \sim-T^{10}\left[\widetilde{\operatorname{Vol}}\left(X_{10}\right)\right]_{r_{\beta}}^{r_{\max }} \sim-T^{9} L^{6}\left(b r_{\max }\right)^{3 / 2}+(L T)^{6}\left(\frac{T}{T_{\mathrm{dec}}}\right)^{9}
$$

In this case, even the term with no cut-off dependence shows pathological behaviour, with an unsuppressed power law $\sim T^{15}$. 
In summary, for $p<5$ the planar specific heat is positive, making possible a gauge theory interpretation of the gravitational thermodynamics. Precisely in this range the first $1 / N^{2}$ or one-loop correction to the free energy scales like a massless free gas in $p$ spatial dimensions. On the other hand, for $p \geq 5$ the optical volume diverges, and we pick a term at $r=r_{\max }$ with ten-dimensional temperature dependence $\beta F \sim-T^{9}$, thus leading to a breakdown of holography.

At this point, it should be noted that the reported violation of holography in the $p=5$ case is a rather marginal effect, since the dependence on the ultraviolet cut-off is only logarithmic. It could be that our criterion is too crude in this case, and a more refined criterion would recover $p=5$ as a holographic system.

The phenomenon described here is similar to the one found in conformal AdS backgrounds in [45] and [10]. However, in those cases, the violations of holography where confined to the interior of the $X$-manifold, and in particular the asymptotic region was always holographic. Here, the asymptotic region is the one that shows ten-dimensional scaling.

The natural interpretation of the holography breakdown for $p \geq 5$ would be, as in [46], [14], as a breakdown of the decoupling between throat physics and ten-dimensional gravity in the asymptotically flat region. In other words, the procedure of blowing up the throat, which is, according to [1], dual to the decoupling of string thresholds in the D-brane theory, is not stable under quantum corrections in the gravity description.

\section{Open-String Thresholds on the Brane}

In the previous sections, we have analyzed some aspects of the thermodynamics of the SYM/SUGRA correspondence, in the strict low energy limit of [1]. From the point of view of the weakly coupled description on the brane, this means that the open-string oscillator modes are decoupled and one only keeps those degrees of freedom corresponding to SYM theory in $p+1$ dimensions. From the point of view of the brane geometries, the same procedure isolates the throat geometries or $X$-manifolds. In particular, these geometries can be continued past the 'neck position' at $r \sim b$. The U.V./I.R. correspondence [1], [47] implies that the radial coordinate acquires the interpretation of a renormalization group scale. Therefore, the 'elimination' of the neck by the blow-up procedure is interpreted as 
a tool to complete the system in the ultraviolet using only Yang-Mills degrees of freedom, wherever that can be done.

It seems natural to suspect that, keeping the neck in place at radial coordinates of order $r \sim b$, should be somehow related to the introduction of the string scale threshold of the brane theory. A precise statement in this direction was made in [16], where corrections to conformality induced by Dirac-Born-Infeld terms (DBI) in the open-string effective action where related to the precise matching of wave functions at the finite position of the neck in the full D3-brane metric.

We can understand this in very general terms via a simple modification of the scaling argument of section 4, indicating that the neck position at $r \sim b$ is giving us some information about $\alpha^{\prime}$-suppressed (i.e. DBI-type) processes in the $D p$-brane theory at large $g_{s} N$. If, instead of the scaled-up geometries $X_{\mathrm{bh}}$ and $X_{\mathrm{vac}}$, we use the complete $E D p$ and $B D p$ metrics in (2.2) and (2.13), we cannot completely scale out the charge radius $b$ from the metric. The functions left undetermined by the scaling argument, such as the one appearing in eq. (4.7), become functions of two variables, i.e. $f\left(z^{2 \alpha} / z_{0}^{2 \alpha}, z^{2 \alpha} / b^{2 \alpha}\right)$ or, translating back to the radial variable $r: f\left(r^{7-p} / r_{0}^{7-p}, r^{7-p} / b^{7-p}\right)$. The result is that each term in (4.9) is multiplied by an unknown function of the ratio $r_{0}^{7-p} / b^{7-p}$.

If we now do a power-expansion in this ratio, the $k$-th order term scales like

$$
\left(\frac{r_{0}}{b}\right)^{k(7-p)}=\left(\alpha^{\prime 2} M_{\mathrm{mag}}^{4}\right)^{k}
$$

where $M_{\mathrm{mag}}$ is the 'magnetic mass gap' in eq. (5.15). We see that the cancellation (4.12) of the $\alpha^{\prime}$ dependence in favour of gauge theory quantities is not complete. Indeed, the leading correction scales like $M_{\mathrm{mag}}^{4}$, i.e. the correct one for the expectation value of the $F^{4}$ combination in the DBI action.

In this section, we adapt the SUGRA thermodynamical analysis of the previous sections to the case where one keeps the neck in place. We also take a peek into the Hagedorn regime of open strings on the $D p$-brane at weak coupling, and obtain $p=5$ as a critical dimension also from this point of view. 


\subsection{Keeping the 'Neck' in Sight}

The main thermodynamical effect of keeping the neck of the brane geometries in place is clearly seen in fig. 1 . Unlike $X_{\mathrm{bh}}$, the full black-brane geometry $B D p$ has always a Schwarzschild regime with negative specific heat and $r_{0} \sim \beta$. This means that, at a temperature of order $T_{b} \sim b^{-1}$, the canonical free energy of $B D p$, with $p<5$, becomes positive, and is therefore suppressed in the canonical ensemble. In the approximation

of smooth geometry, we have then a first-order transition to a high temperature regime dominated by the extremal $D p$-brane geometry $E D p$. In this approximation, there is no $\mathcal{O}\left(N^{2}\right)$ entropy in the high temperature phase, and one has exactly the same behaviour as in [6], [7], with the roles of high and low temperature interchanged.

However, including the effects of thermal winding modes, we know that $E D p$ acquires a cut-off at the Hagedorn radius $r_{\beta}$, generating a stretched horizon and thus a planar entropy. Therefore, the picture of the 'phases', as presented in figs. 4-7, changes simply by the addition of a $X_{\mathrm{Hag}}$-dominated phase at high temperatures, above the line $T=b^{-1}$. We see that one can obtain 'Hagedorn exposure' at sufficiently high temperatures in the gravitational description, provided the throat neck is kept in place. This description can be continued up to string scale temperatures. When $T \sim T_{\mathrm{Hag}}$, the Hagedorn radius becomes of the order of the neck size, $r_{\beta} \sim b$, and the stretched horizon comes out of the throat. At this point it is difficult to imagine any consistent decoupling between the ten-dimensional bulk and the world-volume physics.

On the other hand, for $p \geq 5$ we have $X_{\text {Hag }}$ dominance already in the blow-up limit, and no particular change of the planar thermodynamics takes place by limiting the end of the throat in the ultraviolet regime.

To conclude this discussion, we should remind the reader that we are considering the canonical ensemble throughout the paper. In a microcanonical description, the Hagedorn cut-off $E D p$ metric never dominates over $B D p$, because their masses can only be matched when $r_{0} \sim r_{\beta}$, that is, at the transition line to the SYM description. Indeed, the microcanonical description of the system with the neck in place is equivalent to the one given in [26]. 


\subsection{Hagedorn Regime at Weak Coupling and $p=5$ Again}

Let us consider now the problem of the Hagedorn transition at weak coupling, using more traditional methods. It was mentioned at the end of section 5 that, for $g_{s} N \ll 1$, the radius $r_{\beta}$ tends to be much larger than $r_{0}$ and one can think that the BKT phase transition takes place before $X_{\mathrm{bh}}$ starts to dominate. Of course the whole approach of comparing smooth manifolds breaks down, as the $\alpha^{\prime}$ corrections become of order one everywhere. This is indeed the regime where D-brane perturbation theory is a good approximation. It will be interesting to see if in this approach we can see that $p=5$ is special again.

In the limit $g_{s} \rightarrow 0$, with $g_{s} N$ fixed and small, we can decouple the $D p$-brane worldvolume from closed strings in the ten-dimensional flat bulk space-time, at all energies, i.e. with no assumptions on the value of $\alpha^{\prime}$. In this limit, the bare mass of the $D p$-branes also diverges, and we can neglect brane creation processes in the thermal gas, i.e. we focus on the open-string thermal gas confined in the $p+1$ dimensions of the world-volume. The one-loop free energy of open strings stuck at the $D p$-brane takes the form

$$
\beta F=-\frac{1}{2} \int_{0}^{\infty} \frac{d t}{t} \operatorname{Tr}_{\mathcal{H}_{\text {open }}}(-1)^{F} e^{-t \Delta_{\text {open }}}
$$

with $\mathcal{H}_{\text {open }}$ denoting the open string Hilbert space and the free inverse propagator

$$
\frac{1}{\alpha^{\prime}} \Delta_{\text {open }}=\left(\vec{p}_{N}\right)^{2}+\frac{4 \pi^{2} n^{2}}{\beta^{2}}-\frac{a}{\alpha^{\prime}}+\text { osc. }
$$

Here $\vec{p}_{N}$ is the momentum in the $p$ spatial Neumann directions and $n$ is the quantum of Matsubara frequency. The constant $a$ is the normal ordering intercept: $a=1$ for bosonic strings, while $a_{\mathrm{NS}}=1 / 2$ for Neveu-Schwarz spin structure and $a_{\mathrm{R}}=0$ for the Ramond sector, in the case of superstrings. The Hagedorn behaviour in this representation comes from the ultraviolet region of the open string world-sheet $t \sim 0$, and it is governed by the asymptotic growth of the density of states. We can isolate it by performing a modular transformation to the closed string channel. In this case the previous annulus amplitude becomes a closed string cylinder representing a propagator between boundary states [48].

$$
\beta F(\beta)=-\left\langle B, x\left|P_{\mathrm{GSO}} \frac{1}{\Delta_{\text {closed }}}\right| B, x\right\rangle .
$$

Now the closed string inverse propagator is

$$
\frac{2}{\alpha^{\prime}} \Delta_{\text {closed }}=\vec{p}^{2}+\frac{4 \pi^{2} n^{2}}{\beta^{2}}+\frac{\beta^{2} \ell^{2}}{4 \pi^{2} \alpha^{\prime 2}}-2 \cdot \frac{a+\bar{a}}{\alpha^{\prime}}+\text { osc. }
$$


In this case, $\vec{p}$ explores all spatial dimensions, $n$ is as before the Matsubara frequency, now of the closed strings, and $\ell$ is the thermal winding number of the closed strings. The boundary state $|B, x\rangle$ contains an oscillator part that enforces the Neumann or Dirichlet boundary conditions depending on the coordinate. The zero-mode part is such that the momentum flow is zero for Neumann directions, including the thermal one, and the position is fixed for Dirichlet directions, that is

$$
|B, x\rangle_{\text {zero mode }}=\int d \vec{p}_{D} e^{i \vec{p}_{D} \cdot \vec{x}}\left|\vec{p}_{D} ; \vec{p}_{N}=n=0\right\rangle
$$

Notice that the closed strings are free to wind in the thermal direction (i.e. we sum over $\ell)$.

Using this form of the boundary state we can write the free energy in the closed channel as

$$
\beta F(\beta) \sim-\int d \vec{p}_{D} \sum_{s, \ell} \frac{|\langle B \mid s, \ell\rangle|^{2}}{\frac{\alpha^{\prime}}{2}\left(\vec{p}_{D}^{2}+M_{(s, \ell)}^{2}\right)},
$$

where $s$ is an index for the oscillator states of the closed strings and $M_{(s, \ell)}$ is the mass of closed string states in the dimensionally reduced theory. The Hagedorn transition is here an infrared effect associated to a new massless mode of the closed channel $M^{2} \sim \beta-\beta_{c}$ at winding number $\ell=1$. The critical inverse temperature is

$$
\beta_{c}^{2}=8 \pi^{2} \alpha^{\prime}(a+\bar{a})
$$

Using a Schwinger proper time representation for the propagator, we need to find the singularity coming from $\tau=\infty$ in

$$
\beta F(\beta) \sim-\int d \vec{p}_{D} \sum_{s, \ell}|\langle B \mid s, \ell\rangle|^{2} \int_{0}^{\infty} d \tau e^{-\tau \frac{\alpha^{\prime}}{2}\left(\vec{p}_{D}^{2}+M_{(s, \ell)}^{2}\right)} .
$$

Doing the $\vec{p}_{D}$ integral and picking only the infrared part, we are led to

$$
\beta F(\beta)_{\text {sing }} \sim \int_{1}^{\infty} \frac{d \tau}{\tau^{\frac{9-p}{2}}} e^{-\tau \cdot \frac{C}{\sqrt{\alpha^{\prime}}}\left(\beta-\beta_{c}\right)} \sim\left(\frac{\beta-\beta_{c}}{\sqrt{\alpha^{\prime}}}\right)^{\frac{7-p}{2}} \Gamma\left((p-7) / 2 ; C\left(\beta-\beta_{c}\right) / \sqrt{\alpha^{\prime}}\right) .
$$

Before analyzing this expression for free energy let us show that it simply describes the free energy of a gas of particles moving in a $p+1$ dimensional space-time with the Hagedorn spectrum

$$
\rho(m) \sim m^{-9 / 2} \exp \left(\beta_{c} m\right)
$$


This is the spectrum of open superstrings on a $p$-brane. Calculating the free energy for bosons one gets

$$
\begin{aligned}
-\beta F_{b}=\ln Z_{b} & =-\frac{L^{p}}{(2 \pi)^{p}} \int_{m_{0}}^{\infty} d m \rho(m) \int d^{p} k \log \left[1-\exp \left(-\beta \sqrt{k^{2}+m^{2}}\right)\right] \\
& =\frac{L^{p}}{(2 \pi)^{p}} \int_{m_{0}}^{\infty} d m \rho(m) \sum_{n=1}^{\infty} \frac{1}{n} \int d^{p} k \exp \left(-n \beta \sqrt{k^{2}+m^{2}}\right)
\end{aligned}
$$

and for fermions

$$
\begin{aligned}
-\beta F_{f}=\ln Z_{f} & =\frac{L^{p}}{(2 \pi)^{p}} \int_{m_{0}}^{\infty} d m \rho(m) \int d^{p} k \log \left[1+\exp \left(-\beta \sqrt{k^{2}+m^{2}}\right)\right] \\
& =\frac{L^{p}}{(2 \pi)^{p}} \int_{m_{0}}^{\infty} d m \rho(m) \sum_{n=1}^{\infty} \frac{(-1)^{n+1}}{n} \int d^{p} k \exp \left(-n \beta \sqrt{k^{2}+m^{2}}\right)
\end{aligned}
$$

where $m_{0}$ is the infrared cut-off, which is usually of the same order of magnitude as $\beta_{c}$. Because we are looking at $\beta \sim \beta_{c}$, practically all particles can be considered as non-relativistic ones and we can rewrite $\exp \left(-n \beta \sqrt{k^{2}+m^{2}}\right)$ as $\exp (-n \beta m-$ $\left.n \beta k^{2} / 2 m\right)\left(1+O\left(n \beta k^{4} / m^{3}\right)\right)$; it is easy to check that the neglected terms are of order $(n \beta m)^{-1} \ll 1$. Integrating over $k$ we finally get

$$
-\beta F_{b(f)}=\frac{L^{p}}{(2 \pi)^{p / 2} \beta^{d / 2}} \int_{m_{0}}^{\infty} d m m^{p / 2} \rho(m) \sum_{n=1}^{\infty} \frac{(-1)^{n+1}}{n^{p / 2+1}} \exp (-n \beta m) .
$$

It is clear that in the vicinity of the Hagedorn temperature $\beta \rightarrow \beta_{c}$ one can neglect all terms in the sum with $n \geq 2$ (they will give singularities at $T=n T_{c}$ ) and there is no difference between bosons and fermions in the leading singular term with $n=1$. After integrating over $m$ we get the same answer

$$
\beta F(\beta)_{\text {sing }} \sim\left(\frac{\beta-\beta_{c}}{\sqrt{\alpha^{\prime}}}\right)^{\frac{7-p}{2}} \Gamma\left((p-7) / 2 ; C\left(\beta-\beta_{c}\right) / \sqrt{\alpha^{\prime}}\right),
$$

where the constant $C$ is related to the mass parameter $m_{0}$. Let us note that these two approaches are dual because in the first case we integrated over momenta in the Dirichlet directions and in the second case over the momenta in the Neumann directions (along the brane). For further discussion of such thermal duality properties see [49]. Now one can see that for $p / 2>7 / 2$ the free energy has a power singularity when $\beta \rightarrow \beta_{c}$, whereas in the case $7 / 2>p / 2$ it is finite at $\beta=\beta_{c}$. For $p=7$ one has a logarithmic singularity 
$\beta F \sim \ln \left(\left(\beta-\beta_{c}\right) m_{0}\right)$. That is, for $p \geq 7$ the critical behaviour is similar to the case of normal open strings, in that all thermodynamical functions diverge at the critical point. For $p<7$ the free energy is finite at the transition. The internal energy diverges for $p \geq 5$ and the specific heat diverges for $p \geq 3$.

This means that the Hagedorn temperature starts being 'maximal' for $p=5$, as one has to supply an infinite amount of energy to raise the temperature to that level. However, when the free energy stops diverging, the canonical and microcanonical ensembles could lose equivalence. This is related to the possibility that the specific heat becomes negative. The classic analysis of ref. [50] can be generalized to our case, and one obtains, in terms of the energy density $E / L^{p} \equiv \varrho$, regimes of instability and negative specific heat for $p<7$.

If $p<5$, the microcanonical and canonical ensembles agree for low energy density, $\varrho \ll \varrho_{s}$, with $\varrho_{s}=\left(\ell_{s}\right)^{-p-1}$ the string scale density. On the other hand, for high density, $\varrho \gg \varrho_{s}$, the specific heat is negative and we enter the long-string phased, analogous to the standard behaviour of closed strings in infinite volume. If we agree to call 'Hagedorn regime' the one with super-stringy energy density, we find that the Hagedorn regime of open strings on the $p<5$ brane is similar to the one of closed strings and typical of gravitational systems.

For $p=5$ the condition for the canonical and microcanonical ensembles to be equivalent is

$$
\frac{\varrho}{\varrho_{s}} \ll \log \left(\frac{\varrho}{\varrho_{s}}\right)+\log \left(\frac{L}{\ell_{s}}\right) .
$$

where $L$ is the length scale of the spatial world-volume. This is always satisfied for macroscopic volumes provided we keep the energy density fixed in the thermodynamical limit. So, very dense systems at finite volume would be unstable and get negative specific heat, but this does not occur for sufficiently large world-volume.

For $p=6$ the condition of equivalence of ensembles becomes

$$
\frac{\varrho}{\varrho_{s}} \ll\left(\frac{L}{\ell_{s}}\right)^{p},
$$

which is also easily attainable for sufficently large volume. For $p \geq 7$ the canonical and microcanonical ensembles are equivalent without restrictions.

7 It is unclear whether the long open strings attached to the brane should be thought of as tracing a random walk in the world-volume, or exploring the full ten-dimensional ambient space. 
Thus, the microcanonical analysis seems to be in agreement with the view that $p=5$ is the critical dimension above which (at least for large world-volume) the Hagedorn regime has a 'standard' thermodynamics. This critical dimension has been singled out by both considerations of one-loop holography, and the Hagedorn cernsorship tests. This coincidence suggests that the dualities of [1] may have a generalization to extreme situations, such as stringy energy densities. The main obstacle, in implementing any such scenario, might be the apparent ten-dimensional behaviour that one finds when the supergravity description is pushed to very high temperatures. When the temperature at the 'neck' is of the order of the string scale, the full throat is cut-off by Hagedorn effects, and there seems to be no effective way of separating the bulk ten-dimensional dynamics from the "worldvolume' dynamics. The considerations in this section, showing that, at very weak coupling, $D p$-branes with $p<5$ have a Hagedorn phase similar to that of closed strings, could suggest that these two systems are related to each other without further phase transitions. Roughly speaking, the highly excited long strings would be random walks in ten-dimensions, even if their end-points are confined on the $p+1$-dimensional world-volume.

Even if we keep $N$ finite, and consider a weak, though finite, coupling between the open strings on the brane and the closed strings in the bulk, the thermodynamics of the combined system may be rather non-trivial. Since the Hagedorn temperature can be either limiting or achievable for different branes, depending on $p$, the whole system either may reach the phase transition point, or (if $p \geq 5$ ) the brane will take all the energy from the bulk and stop the whole system from reaching the Hagedorn temperature. Other interesting situations can occur when we have branes inside branes. We will not discuss this subject further here, and we hope to return to it in a future publication.

\section{Discussion}

One of the advantages of considering the supergravity limit of the AdS/CFT correspondence is the access it allows to some non-perturbative information concerning the systems. This is not yet readily available in more direct stringy framework. In this note we have attempted to extract non-perturbative aspects related to the existence of stringy scales. We have done that by both arranging circumstances in which the system is challenged to exhibit its non-perturbative nature at string scales, and also by searching for signs that the correspondence contains its own limitations. 
By considering a toroidal space-time world-volume for the appropriate field theory, we have set up a situation where the corresponding supergravity manifolds contain space and time string scales for low values of the field-theory temperature. We have then motivated the way in which we suggest that dynamical effects modify the manifolds at the string scale. Manifolds containing string-scale temporal loops were capped by a "stretched horizon", while T-duality considerations were applied to those containing small spatial loops. Based on this qualitative picture we have analyzed the consequences of the correspondence. For the "vacuum" manifolds with stringy temporal loops, one could have expected Hagedorn effects to take place locally in the throat part of the manifold. Moreover, that opened the possibility that Hagedorn behaviour of closed strings could have been computable on the gauge theory side. We have found however that smooth black-hole manifolds containing no string scales are favoured upon the "vacuum" manifolds, and thus they censor the probing of the Hagedorn regime.

In string theory, one would imagine that the string would be asked to propagate perturbatively on the "vacuum" manifold and thus the censorship could indicate that in these special backgrounds the Hagedorn transition is just not there, once the fully fledged non-perturbative string theory is considered. This picture could be reinforced by noting that straining the parameters in a manner that the Hagedorn stretched horizon does dominate over the standard smooth horizon, leads at the same time to a spatial manifold with a large bulk curvature (appropriate to the string scale.) The string spectrum in such a manifold cannot be estimated by semi-classical methods, and eventually one would presumably find that the Hagedorn behaviour sets in when the boundary temperature itself is again of the string scale. We have also presented a scaling argument showing that, on the supergravity side, the string scale enters the physics only in that combination that can be re-expressed in terms of the coupling of the gauge theory.

Manifolds with smooth bulk curvature and Hagedorn thresholds can dominate thermodynamics only for $p>4$, but in those cases we have shown that holography breaks down, (at least in a naive sense,) and it is difficult to localize the system in $p+1$ dimensions. A "conspiracy", taking place in the CFT/AdS correspondence and its cousins is at work. The various closed-string/gravity dynamical features always manage to reproduce non-gravitational, gauge-theory physics, when reinterpreted in terms of the boundary theory. The breakdown of holography for $p>4$ is a case where the system had indicated its own limitations. 
We have searched for further limitations by considering manifolds which are not fully blown-up, but rather contain a finite throat merging into flat ten-dimensional Minkowski space. We have found by the similar scaling arguments mentioned before that, in this case, not all the physics on the supergravity side could be expressed in terms of the gauge coupling. This could mean that, while there is still a correspondence in this situation, it is now a correspondence between such supergravity manifolds and gauge systems containing Dirac-Born-Infeld corrections. The possibility of such open-closed string correspondence is made more feasible by a special feature of the thermodynamics of weakly coupled open strings on D-branes. It turns out that for $p<5$ (once again, $p=5$ being a border-line case,) open strings on Dp-branes have characteristics usually associated with closed strings propagating in the absence of branes: at Hagedorn temperatures they may exhibit a phase transition.

The consideration of the thermodynamics of such manifolds leads to uncovering a rich qualitative structure, some of which could perhaps serve as a basis for a phase diagram of some gravitational systems. It is encouraging that the different regions emerging have their counterparts in the string/black-hole correspondence principle of [26]. The identification of such non-perturbative features directly in a string framework remains a chalenge, and knowing the answer in some cases may be of some help.

\section{Aknowledgements}

We thank D. Birmingham, M.B. Green, D. Kutasov and J.M. Maldacena for useful discussions. The work of E. R. is partially supported by the Israel Academy of Sciences and Humanities-Centres of Excellence Programme, and the American-Israel Bi-National Science Foundation. 


\section{References}

[1] J. Maldacena, "The Large N Limit Of Superconformal Field Theories And Supergravity", hep-th/9711200.

[2] S.S. Gubser, I.R. Klebanov and A.W. Peet, Phys. Rev. D54 (1996) 3915;

I.R. Klebanov, Nucl. Phys. B496 (1997) 217;

S.S. Gubser, I.R. Klebanov and A.A. Tseytlin, Nucl. Phys. B499 (1997) 217;

S.S. Gubser and I.R. Klebanov, Phys. Lett. B413 (1997) 41.

[3] G.W. Gibbons and P.K. Townsend, Phys. Rev. Lett. 71 (1993) 5223;

G.W. Gibbons, G.T. Horowitz and P.K. Townsend, Class. Quant. Grav. 12 (1995) 297.

[4] M. R. Douglas, J. Polchinksi and A. Strominger, J. High Energy Phys. 12 (1997) 003, hep-th/9703031.

[5] S.S. Gubser, I.R. Klebanov and A.W. Peet, Phys. Rev. D54 (1996) 3915, hepth/9602135.

[6] E. Witten, "Anti De Sitter Space and Holography", hep-th/9802150.

[7] E. Witten, "Anti-de Sitter Space, Thermal Phase Transition, And Confinement In Gauge Theories", hep-th/9803131.

[8] S. Hawking and D. Page, Commun. Math. Phys. 78B (1983) 577.

[9] G. 't Hooft, "Dimensional Reduction In Quantum Gravity", in Salamfest, Trieste, 1993, p. 284, gr-qc/9310026;

J.D. Bekenstein, Phys. Rev. D49 (1994) 1912;

L. Susskind, J. Math. Phys. 36 (1995) 6377, hep-th/9409089.

[10] J.L.F. Barbón and E. Rabinovici, "Extensivity Versus Holography in Anti-de Sitter spaces", hep-th/9805143;

E. Rabinovici, http://www.itp.ucsb.edu/online/strings98/rabinovici/

[11] A. Giveon, D. Kutasov and N. Seiberg, "Comments on String Theory on AdS 3 ", hep-th/9806194.

[12] D. Birmingham, "Topological Black Holes in Anti-de Sitter Space," hep-th/9808032.

[13] N. Itzhaki, J. Maldacena, J. Sonnenschein, and S. Yankielowicz, Phys. Rev. D58 (1998) 046004, hep-th/9802042.

[14] J. Maldacena and A. Strominger, J. High Energy Phys. 12 (1997) 008, hepth/9710014.

[15] G. Horowitz and A. Strominger, Nucl. Phys. B360 (1991) 197;

M.J. Duff, R.R. Khuri and J.X. Lu, Phys. Rep. 259 (1995) 213, hep-th/9412184. 
[16] S.S. Gubser, A. Hashimoto, I.R. Klebanov and M. Krasnitz, Nucl. Phys. B526 (1998) 393 , hep-th/9803023.

[17] S. de Alwis, "Supergravity, the DBI Action and Black Hole Physics", hep-th/9804019.

[18] I.R. Klebanov and A.A. Tseytlin, Nucl. Phys. B475 (1996) 164, hep-th/9604089.

[19] F. Tangherlini, Nuovo Cimento 77 (1963) 636;

R. Myers and M. Perry, Ann. Phys. 172 (1986) 304.

[20] T. Banks and M.B. Green, J. High Energy Phys. 05 (1998) 002, hep-th/9804170;

M. Bianchi, M.B. Green, S. Kovacs and G. Rossi, "Instantons in Supersymmetric Yang-Mills and D-Instantons in IIB Superstring Theory", hep-th/9807033.

[21] I.I. Kogan and G. Luzón, 'D-Instantons on the Boundary", hep-th/9806197.

[22] C.-S. Chu, P.M. Ho and Y.-Y. Wu, "D-Instanton in AdS $S_{5}$ and Instanton in $S Y M_{4}$ ", hep-th/9806103.

[23] S.S. Gubser, I.R. Klebanov and A.A. Tseytlin, 'Coupling Constant Dependence in the Thermodynamics of $N=4$ Supersymmetric Yang-Mills Theory", hep-th/9805156.

[24] E. Witten, “Theta Dependence In The Large $N$ Limit Of Four Dimensional YangMills Theories", hep-th/9807109.

[25] R. Gregory and R. Laflamme, Phys. Rev. Lett. 70 (1993) 2837, hep-th/9301052.

[26] G.T. Horowitz and J. Polchinski, Phys. Rev. D55 (1997) 6189, hep-th/9612146.

[27] S. Das and S. Mathur, Phys. Lett. B365 (1996) 79, hep-th/9601152;

J.M. Maldacena and L. Susskind, Nucl. Phys. B475 (1996) 679, hep-th/9604042.

[28] D.J. Gross and E. Witten, Phys. Rev. D21 (1980) 446.

[29] L. Susskind, 'Matrix Theory Black Holes and the Gross-Witten Transition", hepth/9805115.

[30] G. Polhemus, Phys. Rev. D56 (1998) 2202, hep-th/9612130.

[31] T. Banks, W. Fishler, I.R. Klebanov and L. Susskind, Phys. Rev. Lett. 80 (1998) 226, hep-th/9709091, J. High Energy Phys. 01 (1998) 008, hep-th/9711005.

[32] G. Horowitz and E. Martinec, Phys. Rev. D57 (1998) 4935, hep-th/9710217.

[33] J.M. Maldacena, Phys. Rev. Lett. 80 (1988) 4859, hep-th/9803002;

S-J Rey and J. Yee, "Macroscopic Strings as Heavy Quarks in Large $N$ Gauge Theory and Anti-de Sitter Supergravity", hep-th/9803001.

[34] A. Brandhuber, N. Itzhaki, J. Sonnenschein and S. Yankielowicz, "Wilson Loops in the Large N Limit at Finite Temperature," hep-th/9803137;

S-J. Rey, S. Theisen and J-T. Yee, Nucl. Phys. B527 (1998) 171, hep-th/9803135. 
[35] D.J. Gross and H. Ooguri, "Aspects of Large N Gauge Dynamics as Seen by String Theory," hep-th/9805129.

[36] A. Brandhuber, N. Itzhaki, J. Sonnenschein and S. Yankielowicz, J. High Energy Phys. 06 (1998) 001 , hep-th/9803263.

[37] I.I. Kogan, JETP. Lett. 45 (1987) 709;

B. Sathiapalan, Phys. Rev. D35 (3277.)

[38] V. Berezinski, JETP. Lett. 32 (1971) 493;

J. Kosterlitz and D. Thouless, J. Phys. C6 (1973) 1181.

[39] J. Atick and E. Witten, Nucl. Phys. B310 (1988) 291.

[40] N. Mavromatos, "World-sheet Defects, Strings and Quark Confinement," hepth/9803189.

[41] L. Susskind, "Some Speculations about Black Hole Entropy in String Theory", hepth/9309145;

A. Sen, Mod. Phys. Lett. A10 (1995) 2081, hep-th/9504197;

G. Horowitz, "The Origin of Black Hole Entropy in String Theory", gr-qc/9604051.

[42] M. Li, "Evidence for Large $N$ Phase Transition in $N=4$ Super Yang-Mills Theory at Finite Temperature", hep-th/9807196.

[43] G. 't Hooft, Nucl. Phys. B256 (1985) 727.

[44] G. W. Gibbons and M. J. Perry, Proc. R. Soc. Lond. A358, (1978), 467 ;

J.S. Dowker and G. Kennedy, J. Phys. A11 (1978) 895.

[45] G. T. Horowitz and H. Ooguri, Phys. Rev. Lett. 80 (1998) 4116, hep-th/9802116.

[46] A. Sen, Adv. Theor. Math. Phys. 2 (1998) 51, hep-th/9709220;

N. Seiberg, Phys. Rev. Lett. 79 (1997) 3577, hep-th/9710009.

[47] L. Susskind and E. Witten, "The Holographic Bound In Anti-de Sitter Space," hepth/9805114.

[48] M.B. Green, Phys. Lett. B266 (1991) 325, Phys. Lett. B329 (1994) 435, hepth/9403040.

[49] M.A. Vázquez-Mozo, Phys. Lett. B388 (1996) 494, hep-th/9607052.

[50] S. Frautschi, Phys. Rev. D3 (1971) 2821;

R.D. Carlitz, Phys. Rev. D5 (1972) 3231. 